\title{
DIAGNOSTIC AND PROGNOSTIC SIGNIFICANCE OF POISONING SEVERITY SCORE, GLASGOW COMA SCALE, VITAL SIGNS, AND ENZYMATIC BIOMARKERS IN CASES WITH ACUTE ANTICHOLINESTERASE INSECTICIDES POISONING
}

\author{
Mohamed F. Khodeary ${ }^{1,2}$ and Shereen M. S. Elkholy ${ }^{1}$ \\ ${ }^{1}$ Department of Forensic Medicine and Clinical Toxicology, Faculty of Medicine, Benha \\ University \\ ${ }^{2}$ Benha Poison Control Unit, Benha University Hospitals
}

\begin{abstract}
Introduction: Organophosphate (OPC) and carbamate (CMC) compounds are highly toxic anticholinesterase (AntiChE) pesticides, extensively used worldwide, and still responsible for poisoning epidemics. Multi-organ dysfunctions have been reported following AntiChE poisoning. Aim: This study aimed to assess the correlation between poisoning severity score (PSS), Glasgow coma scale (GCS), and butyrylcholinesterase (BuChE) levels with clinical and laboratory changes in adult patients with acute AntiChE intoxication. Subjects and methods: This study included 25 individuals in healthy-control-group (HC-group) and 75 patients in AntiChE-group. According to poisoning severity criteria, patients were allocated into mild, moderate, or severe intoxicated-group. Gender, age, and causative substance data were reported. Clinical parameters like grade of PSS, delay in hospital arrival time (DHA), vital signs functions, GCS score, and length of hospital stay (LHS) were measured. The blood levels of biochemical parameters ( $\mathrm{pH}$, sodium, potassium, and random blood glucose) and enzymatic biomarkers (BuChE, cardiac creatine kinase-myocardial band and cardiac troponin I, pancreatic amylase and lipase, hepatic aspartate and alanine aminotransferases, and kidney urea and creatinine) were estimated. Results: Cases were mostly females, aged 23.82 \pm 0.82 -year, and intoxicated by OPC, especially malathion. The commonest clinical findings were minor manifestations of PSS, DHA for 2-hour, drowsy GCS level, and relatively short LHS for $\leq 24$-hour, while vital signs abnormalities predominantly included tachycardia, hypertension, tachypnea, and hyperthermia. The main biochemical abnormalities were metabolic acidosis, hypernatremia, hypokalemia, and hyperglycemia. Low BuChE levels were detected in all cases, whereas increased enzymatic biomarkers levels were noticed in some cases. On admission, the proportions and mean values of overall clinical and laboratory parameters showed statistically significant differences among the three intoxicatedgroups and between AntiChE-group and HC-group. Additionally, PSS grade, GCS score, and degree of BuChE inhibition significantly correlated with DHA, all vital signs, LHS, and overall laboratory parameters as well as between each other. At discharge, the initial clinical and laboratory abnormalities were markedly improved and showed statistically insignificant differences from HC-group except for BuChE levels remained significantly low. Conclusion: Although the PSS, GCS, and BuChE seem similarly useful clinical indices at predicting severity of AntiChE poisoning, however, the efficacy of PSS outperform the GCS and BuChE effectiveness.
\end{abstract}


Keywords: Anticholinesterase poisoning, Organophosphate and Carbamate Intoxication, Clinical and Laboratory Abnormalities, Poisoning Severity Score, Glasgow Coma Scale, Predicting Severity of AntiChE Poisoning

\section{INTRODUCTION}

Pesticide compounds include several diverse groups or mixtures of chemicals which play an important economic role in preventing, destroying, or controlling a broad spectrum of potentially hazardous pests. The most widely used classes of pesticides are organophosphates, carbamates, and pyrethrins or pyrethroids compounds (Erdman, 2004).

Organophosphate compounds (OPC) and carbamate compounds (CMC) are spontaneously degradable anticholinesterase (AntiChE) pesticides synthesized to replace the more toxic organochlorine substances which persist and accumulate in the environment (Colović et al., 2013).

The OPC and CMC are extensively utilized all over the world for different agricultural, industrial, and domestic purposes. Despite the development of the less toxic classes of pesticides such as pyrethroids and neonicotinoids, the uses of OPC and CMC in urban settings are still high due to their great efficacy, relatively low cost, and lack of bioaccumulation in the environment (Costa, 2006).

The OPC and CMC are highly toxic acetylcholinesterase-inhibiting pesticides that continue to be intensively responsible for poisoning epidemics, particularly in developing countries (Aslan et al., 2011). The easy availability of such substances commonly produces human toxicity by accidental and suicidal manner (Sungur and Güven, 2001).

Acute poisonings by the AntiChE agents, particularly OPC and CMC, are an important life-threatening clinical problem and commonly responsible for considerable hospital-related admission, morbidity, and mortality worldwide (Sam et al., 2009; Muley et al., 2014). According to the estimated data of the World Health Organization, around three million cases with pesticide poisonings occur each year, resulting in approximately 250,000 to 370,000 annual deaths throughout the world (Goldsmith et al., 2016). In Egypt, a total of 20,300 AntiChEpoisoned cases were recorded over a period of 17-year (1966-1982) with an average of 1194 AntiChE-poisoned individuals/year, while in year 2006 the number of cases was increased; reached approximately 3 times (3564 persons) those occurred annually during the past decades (Mansour and Gamalludin, 2008).

The OPC and $\mathrm{CMC}$ act as irreversible and reversible cholinesterase inhibitors, respectively, causing excessive accumulation of the neurotransmitter acetylcholine within the synaptic cleft with subsequent overstimulation of muscarinic and nicotinic receptors present in the peripheral and central nervous system, producing cholinergic toxidrome (Colović et al., 2013; Lee et al., 2015).

Clinical manifestations of acute toxicity appear within few hours post exposure to AntiChE substances. The classic muscarinic effects comprise sweating, lacrimation, salivation, diarrhea, urination, gastrointestinal discomfort, pin-point pupils, emesis, rhinorrhea, increased bronchial secretion, bronchoconstriction, and dyspnea. The nicotinic effects include 
muscle fasciculations, twitching, weakness and/or paralysis in severe cases. Central nervous system signs include restlessness, tremor, confusion, convulsions, respiratory depression, and coma (Rubinshtein et al., 2002).

Other numerous complications such as disturbances of acid-base equilibrium, electrolyte values, and blood glucose levels have been documented in several cases poisoned with AntiChE family (Kara et al., 2002; Liu et al., 2008; Gündüz et al., 2015).

Cardiovascular toxicity often accompanies poisoning by AntiChE compounds, which may be serious or fatal. Several studies have recorded different patterns of cardiotoxic manifestations and electrocardiographic abnormalities in patients with AntiChE poisoning including disturbances in vital functions and conduction defects. These cardiac disorders commonly occur within few hours after exposure and many physicians may not fully recognize them. Hence, early detection and adequate treatment can potentially prevent this life-threatening condition and improve outcome (Kara et al., 2002; Karki et al., 2004).

Acute pancreatitis is also recognized as an important complication of intoxication by AntiChE agents and has been reported in experimental and humans studies (Singh et al., 2007; Aslan et al., 2011). However, the true incidence of pancreatic injury is unknown and may be more frequent than clinically suspected. Additionally, patients with AntiChE poisonings may present with subclinical traits of acute pancreatitis. Thus, proper clinical and biochemical investigations can help in diagnosis (Sahin et al., 2002; Brahmi et al., 2005).
Although liver has an important role in degradation of AntiChE substances, the effects of these compounds on the liver functions are not well understood. Acute hepatotoxicity as a complication of exposure to these agents has been frequently reported in several studies (Sahin et al., 2002; Singh et al., 2011).

The association between AntiChE poisoning and subsequent risk of development of acute kidney injury has been mentioned in a few numbers of the articles. Misidentification or underestimation of AntiChE-related nephrotoxicity may cause severe adverse outcomes. In a populationbased retrospective cohort study, the incidence of AntiChE-related nephrotoxicity has been higher than in the non-AntiChE poisoned cohort (Lee et al., 2015). Multiple organs distress syndrome, impairment of renal function, and acute renal failure have been reported in human following acute exposure to AntiChE substances that widely correlated with death (Agostini and Bianchin, 2003; Kozacı et al., 2012). Also, exposures to different types of AntiChE substances have been associated with nephrotoxicity in occupational workers (Singh et al., 2011).

\section{AIM OF THE WORK}

The present study was designed to evaluate the correlation between poisoning severity score grades, Glasgow coma scale scores, and butyrylcholinesterase levels with clinical and laboratory findings among acute AntiChE intoxicated patients who admitted to Benha Poison Control Unite. Also, the validity of these parameters in predicting the prognosis and outcome of acute AntiChE poisoning were assessed. 


\section{SUBJECTS \& METHODS}

This prospective study was carried out over a period of 12-month from the $1^{\text {st }}$ of March 2016 to the end of February 2017 at BPCU, Benha University Hospitals, El-Qalyubia, Egypt, after approval from the Research Ethical Committee of Benha Faculty of Medicine. Prior to starting the study, all proposed procedures were explained to the patients or relatives and all participants provided informed verbal consent. All selected patients with only acute AntiChE intoxication that fulfilled the inclusion criteria were enrolled in the present study.

A. Inclusion criteria:

1- History of acute AntiChE ingestion only (OPC and CMC).

2- Characteristic cholinergic toxidrome of cholinesterase inhibitors such as excessive salivation, lacrimation, and sweating, pinpoint pupils, chest wheezes and crepitations or rales, bradycardia, stool and urine incontinence, abdominal pain, nausea, and vomiting, muscular fasciculations or weakness, tachycardia, and manifestations of central nervous system stimulation or depression.

3- Improvement of the cholinergic toxidrome after administration of atropine and oximes.

4- Low BuChE level or activity.

5- Survived patients.

6- Patients or their relatives who gave informed consent.

B. Exclusion criteria:

1- All patients with co-ingestions.

2- All patients who received anticholinergic therapy before commencement of the study.

3- All patients with past medical history of special habits (smoking and intake of drugs, alcohol, or addiction), chronic exposure to anticholinesterase compounds, concomitant chronic systemic diseases (hepatitis, diabetes mellitus, hypertension, psychiatric, epilepsy, immunological, and neuromuscular), and/or any organ dysfunction.

4- All asymptomatic patients with normal BuChE levels and deceased cases.

5- Non-cooperative patients who refused to participate in the study.

Initially, all patients were rapidly stabilized, evaluated clinically, blood sampled, and managed on admission according to a standard clinical protocol of Eddleston et al. (2004).

\section{Subjects:}

All participated individuals were divided as follows:

(I) Healthy control group (HCgroup): The individuals who kindly accepted to participate in this study were recruited randomly from the surrounding community with matched age and gender and matched as closely as possible with the intoxicated cases. They were non-smokers and free of any acute or chronic diseases with normal endocrine, cardio-pulmonary, and hepato-renal systems on clinical examination as well as they had not exposed to cholinesterase inhibitors and did not receive any drug during the last 2 weeks.

(II) Anticholinesterase group (AntiChE-group): Included the total number of cases with both OPC and CMC poisonings. Moreover, all patients were allocated into three intoxicated-groups according to their severity grades of poisoning namely mild, moderate, or severe.

\section{Methods:}

All suggested procedures were accomplished after stabilization of any life-threatening conditions and before initiation of therapy. 


\section{I- The epidemiological data:}

The gender and age of all participants were recorded. Additionally, the subtypes of AntiChE causative compounds were reported.

\section{II- The clinical data:}

Clinical data in terms of the following parameters were carried out for all selected patients:

1- Delay in hospital arrival (DHA) time: time lapse or lags between exposures to AntiChE substances and the time of arrival to the unit.

2- Length of hospital stay (LHS) or the hospitalization period.

3- Vital signs: Each of heart rate (HR), blood pressure (BP), respiratory rate (RR), and body temperature (BT) was measured and documented. The abnormalities of vital functions in adults included bradycardia (HR $<60$ beats/minute), tachycardia (HR $>100$ beats/minute), hypotension (systolic blood pressure; SBP $<90 \mathrm{mmHg}$ and/or diastolic blood pressure; DBP $<60$ $\mathrm{mmHg}$ ), hypertension (SBP >160 $\mathrm{mmHg}$ and/or DBP >95 $\mathrm{mmHg}$ ), bradypnea (RR <12 breaths/minute), tachypnea (RR $>25$ breaths/minute), hypothermia (BT $<36.8{ }^{\circ} \mathrm{C}$ ), and hyperthermia (BT $>37.2{ }^{\circ} \mathrm{C}$ ) (Nelson et al., 2011; Vijayakumar et al., 2011).

4- Severity of poisoning: The clinical severity of each enrolled case was evaluated according to PSS criteria of the European Association of Poisons Centres and Clinical Toxicologists (EAPCCT) (Persson et al., 1998). According to severity grades, the selected patients were classified into 3 groups as follows:

A. Mild (grade 1): Cases with minor, transient, and spontaneously resolving symptoms within 24 hours of exposure.

B. Moderate (grade 2): Cases with pronounced more prolonged symptoms for 24 hours or more after exposure.

C. Severe (grade 3): Cases with life-threatening manifestations.

5- Level of consciousness: The conscious states of all patients were assessed against the criteria of GCS. The scale is composed of three tests: eye (score: 1-4), verbal (score: 1-5), and motor (score: 1-6) responses. The resulting sum of the three points gives each patient a score in the range of 315. The patients' neurologic status were scored as fully awake (15), drowsy (1214), and stupor or coma (3-11) (Baydin et al., 2007).

\section{III- Laboratory investigations:}

During clinical examination, two venous and two arterial blood samples (5 $\mathrm{ml}$ each) were withdrawn from each selected patient, one at admission and the other at discharge. While, in the HC-group, a single venous and single arterial blood samples were taken from each individual. Venous blood samples were withdrawn directly into vacutainers containing coagulants, incubated at $37^{\circ} \mathrm{C}$ for 15 minutes until blood clotted, and then centrifuged for 15 minutes at 3000 revolutions per minute to separate the sera. The clear non-haemolysed supernatant sera were quickly removed, collected in tubes, and immediately stored at $-20^{\circ} \mathrm{C}$ till been investigated for the proposed biochemical parameters. Whereas, arterial blood samples were collected directly into vacutainers containing heparin anticoagulant and sent to the laboratory as soon as possible for $\mathrm{pH}$ analysis. The biochemical levels of $\mathrm{pH}$ of acid-base balance, sodium, potassium, and random glucose as well as the enzymatic biomarkers values of $\mathrm{BuChE}$ and cardiac (creatine kinasemyocardial band and cardiac troponin I), pancreatic (amylase and lipase), hepatic (aspartate aminotransferase and 
alanine transaminase), and kidney (blood urea and creatinine) organs were estimated as follows:

A. Arterial blood pH level was measured spectrophotometrically according to the method of Scott et al. (2006) using EliTech UK IRMA TRUpoint blood gas analyzer (reference value: 7.35-7.45).

B. Serum sodium (Na) and potassium $(\mathrm{K})$ levels were estimated according to the atomic absorption flame photometric method of Pincus and Lifshitz (2007) using the NA-p CAL and K-p CAL reagent kits, respectively, obtained from Spinreact Company (Spain) (reference value of $\mathrm{Na}$ : $135-145 \mathrm{mEq} / \mathrm{L}$; reference value of $\mathrm{K}: 3.5-5.5 \mathrm{mEq} / \mathrm{L})$.

C. Serum random blood glucose (BLG) level was assayed spectrophotometrically according to the method of Kunsst (1994) using hexokinase enzymatic reagent kits purchased from Spinreact Company (Spain) (reference value: 70-140 $\mathrm{mg} / \mathrm{dl})$.

D. Serum BuChE enzyme activity was evaluated spectrophotometrically according to the method of Whittaker et al. (1983) using cholinesterase reagent kits obtained from Spinreact Company (Spain) (reference value: 4850-12.000 U/L).

E. Serum creatine kinasemyocardial band (CK-MB) enzyme activity was quantitatively measured spectrophotometrically according to the procedure of Gerhardt and Waldenström (1979) using anti CK-M immune-inhibition reagent kits obtained from Spinreact Company (Spain) (reference value: $<24 \mathrm{U} / \mathrm{L}$ ).

F. Cardiac troponin I (cTnI) enzyme activity was quantitatively measured according to the method of Adams et al. (1994) using Accu-Bind
cTnI Microplate Enzyme Linked Immunosorbent Assay (ELISA) commercial kits provided from Monobind Inc, Lack Forest (USA) (reference value: $\leq 1.3 \mathrm{ng} / \mathrm{ml}$ ).

G.Serum amylase enzyme activity was estimated according to the amylase-colorimetric method of Foo and Bais (1998) using the chloronitrophenol maltotriose glucose kinetic reagent kits supplied from Spinreact Company (Spain) (reference value: up to $90 \mathrm{U} / \mathrm{L})$.

H.Serum lipase enzyme activity was determined according to the lipasecolorimetric kinetic method of Lorentz (1998) using the lipase-LS dilauryl-racglycero-3-glutaric acid-(6methylresorufin)-ester reagent kits supplied from Spectrum Diagnostics The Egyptian Company for Biotechnology (S.A.E) (reference value: up to $60 \mathrm{U} / \mathrm{L}$ ).

I. Serum aspartate aminotransferase (AST) and alanine transaminase (ALT) hepatic enzymes activities were measured spectrophotometrically according to the method of Pincus et al. (2007) using the AST and ALT NADH kinetic UV IFCC reagent kits, respectively, provided from Spinreact Company (Spain) (reference value of AST: 38 U/L; reference value of ALT: $40 \mathrm{U} / \mathrm{L}$ ).

J. Serum blood urea (BLU) and creatinine (CRE) renal enzymes activities were estimated, respectively, according to urease- and creatininecolorimetric methods of Lamb and Price (2008) using the ureaseglutamate dehydrogenase and Jaffe's sodium picrate reagent kits, respectively, provided from Spinreact Company (Spain) (reference value of BLU: $15-45 \mathrm{mg} / \mathrm{dL}$; reference value of CRE: $0.7-1.4 \mathrm{mg} / \mathrm{dL}$ in males and 0.6$1.1 \mathrm{mg} / \mathrm{dL}$ in females). 


\section{Statistical Methods}

The collected data were defined, coded, and analyzed using the statistical package of social science software version 16 (SPSS Inc, Chicago, IL Company). Categorical data were presented as number and percentages while quantitative data were expressed as mean \pm standard error of measurement (SEM). Chi square test $\left(\mathrm{X}^{2}\right)$, one-way analysis of variance (ANOVA), and Pearson's correlation coefficient were used as tests of significance. Chi square $\left(\mathrm{X}^{2}\right)$ was used as test for comparison between number and percentage of two or more groups to compare frequencies. ANOVA was used to compare between more than two continuous variables and significant results followed by Tukey HSD post-hoc multiple comparisons test to detect which pairs were significant. Pearson's correlation coefficient was used to evaluate the linear association between two quantitative variables, where positive and negative results indicating direct and inverse relationship, respectively. A $\mathrm{P}$-value of $<0.05 \quad(\mathrm{P}<0.05)$ was considered as significant level.

\section{RESULTS}

During this one year prospective study, 75 patients of both sexes with AntiChE poisoning clearly fulfilled the inclusion criteria and accepted study participation.

As shown in Table (1), HC-group included 25 individuals ( 9 males and 16 females with a sex ratio of $1: 1.77$ ) and the AntiChE-group comprised 75 patients (30 males and 45 females with a sex ratio of $1: 1.5$ ). There was more prevalence of acute poisoning among female than male and the mean age of the overall patients was $23.82 \pm 0.82$ years. Statistical analysis showed non- significant differences between HCgroup, AntiChE-group, and Intoxicated-groups regarding the proportions of gender and age variables.

As noted in Table (2), poisoning by OPC was more common than by CMC with malathion and aldicarb were the most frequently utilized subtypes among both substances, respectively.

As illustrated in Table (3), the main bulk of patients in AntiChE-group experienced mild toxic manifestations (grade 1) of PSS, followed in order of frequency by those with moderate (grade 2) and severe (grade 3) toxicities, DHA for 2-hour followed by those with $\geq 5$-hour post exposure, and relatively short LHS for $\leq 24$-hour then for 48-hour.

The vast majority of cases in AntiChE-group showed abnormalities in their HR, BP, RR, and conscious status, while unusual BT were less frequently observed.

The most common findings of clinical abnormalities were tachycardia, hypertension, tachypnea, hyperthermia, and drowsy conscious score of GCS. Chi-square statistical analysis between different proportions of the overall clinical parameters showed significant differences among the intoxicatedgroups.

As observed in Table (4), the mean values of PSS, DHA, GCS, and LHS in AntiChE-group were $1.69 \pm 0.09$, $3.5 \pm 0.32$ hours, $13.1 \pm 0.25$, and $45.65 \pm 4.03$ hours, respectively. Among the Intoxicated-groups, patients with moderate and severe grades of poisoning depicted statistically significant increases in the mean values of DHA and LHS, while their mean values of GCS exhibited statistically significant decreases when compared to the corresponding values of cases with 
mild toxicity grade. Also, both mean values of DHA and LHS were statistically significantly higher and that of GCS was statistically significantly lower in severe than moderate cases.

Moreover, the mean values of $\mathrm{HR}$, diastolic BP (DBP), systolic BP (SBP), $\mathrm{RR}$, and BT in AntiChE-group were $87.32 \pm 3.77$ beats/minute, $74.91 \pm 2.57$ $\mathrm{mmHg}, \quad 118.69 \pm 3.83 \quad \mathrm{mmHg}$, $21.37 \pm 0.84$ breaths/minute, and $37.2 \pm 0.08{ }^{\circ} \mathrm{C}$, respectively. The mean values of all vital signs were statistically significantly increased and decreased in moderate and severe poisonings, respectively, as compared to the corresponding values of mild toxicity grade. Also, the differences in vital signs were statistically significantly reduced in severe than moderate poisonings.

As recognized in Table (5), statistical analysis revealed significant elevations in the total mean values of $\mathrm{HR}$ and $\mathrm{RR}$ and significant reduction in the total mean value of GCS score of AntiChE-group on admission when matched with those of HC-group. Also, statistically significant differences in the mean values of all disturbed vital signs and conscious status were observed between AntiChE-group on admission and HC-group.

On the other hand, these initial changes in the mean values of the total and abnormal clinical findings showed marked improvement at discharge as evidenced by statistically insignificant and significant distinctions from the mean values of HC-group and AntiChE-group at admission, respectively.

As detected in Table (6), the rvalues of DHA and LHS showed statistically significant strong positive correlation with PSS grades, however, significant strong negative correlation were observed between PSS grades and the total and slow HR, total, low, and high DBP, low and high SBP, low BT, GCS score, and BuChE enzyme. Moreover, there were significant moderate negative correlation between PSS grades and the other statistically analyzed variables.

The r-values of DHA and LHS showed statistically significant strong negative association with GCS scores; nonetheless, significant strong positive association were noticed between GCS scores and slow and rapid HR, low DBP, low SBP, slow and rapid RR, low $\mathrm{BT}$, and BuChE enzyme. Additionally, there were significant moderate positive association between GCS scores and the other statistically analyzed variables.

The r-values of DHA and LHS depicted statistically significant strong negative relationship with $\mathrm{BuChE}$ enzyme, additionally; there were statistically significant strong positive relationship between $\mathrm{BuChE}$ enzyme and total HR and total and slow RR. Furthermore, there were significant moderate positive relationship between BuChE enzyme and the other statistically analyzed variables.

As manifested in Table (7), the main reported abnormal biochemical findings in AntiChE-group were low $\mathrm{pH}(30 ; 40 \%)$, hypernatremia (21; $28 \%)$, hypokalemia $(42 ; 56 \%)$, and hyperglycaemia $(43 ; 57.33 \%)$, whereas the chief recorded abnormal laboratory findings of enzymatic biomarkers were low BuChE $(75 ; 100 \%$ and the activity was decreased by $50 \%$ in $32 ; 42.67 \%$ cases) and high serum levels of CK-MB (28; 49.12\%), cTnI $(18 ; 24 \%)$, amylase $(25 ; 33.33 \%)$, lipase $(20 ; 26.67 \%)$, AST (41; 54.67\%), ALT (33; 44\%), BLU $(33 ; 44 \%)$, and CRE $(29 ; 38.67 \%)$. Chisquare statistical analysis between 
different proportions of the overall laboratory parameters showed significant differences among the intoxicated-groups.

As delineated in Table (8), in moderate and severe intoxicated-groups on admission, the mean values of $\mathrm{pH}$, $\mathrm{K}$, and BuChE enzyme were statistically significantly decreased, while those of Na, BLG, CK-MB, cTnI, amylase, lipase, AST, ALT, BLU, and CRE were statistically significantly increased when compared with the corresponding values of mild intoxicated-group.

As evidenced in table (9), in AntiChE-group on admission, statistical analysis depicted significant decreases in the mean values of $\mathrm{pH}, \mathrm{K}$, and BuChE enzyme, whereas those of $\mathrm{Na}$, BLG, CK-MB, cTnI, amylase, lipase, AST, ALT, BLU, and CRE exhibited significant increases when compared with the corresponding values of HCgroup.

Otherwise, these initial alterations in the mean values of almost all investigated biochemical and enzymatic biomarkers were remarkably returned back to normal levels at discharge as evidenced by statistically insignificant and significant disparities from the mean values of HC-group and AntiChE-group at admission, respectively. However, comparison between the mean values of $\mathrm{BuChE}$ enzyme of AntiChE-group at discharge and at admission revealed statistically significant increase, but still significantly lowers than HC-group value.

As demonstrated in Table (10), the r-values of all investigated biochemical and enzymatic biomarkers of AntiChEgroup were statistically significantly correlated with PSS grades. There was a statistically significant strong negative correlation between $\mathrm{pH}$ and PSS scores, whereas the r-values of $\mathrm{Na}$, $\mathrm{K}, \mathrm{BLG}, \mathrm{CK}-\mathrm{MB}, \mathrm{cTnI}$, AST, and ALT showed statistically significant strong positive correlations with PSS scores. In addition, the r-values of amylase, lipase, BLU, and CRE showed statistically significant moderate positive correlations with PSS scores.

The r-values of all estimated biochemical and enzymatic biomarkers of AntiChE-group were statistically significantly associated with GCS scores, wherein the r-values of $\mathrm{pH}$ and $\mathrm{K}$ showed statistically significant moderate positive associations, those $\mathrm{r}$ values of $\mathrm{Na}$, BLG, CK-MB, cTnI, AST, ALT, BLU, and CRE depicted statistically significant moderate negative associations, and those $\mathrm{r}$ values of amylase and lipase showed statistically significant weak negative associations.

The r-values of all assayed biochemical and enzymatic biomarkers of AntiChE-group were statistically significantly associated with $\mathrm{BuChE}$ enzyme, wherein the r-values of CKMB and cTnI revealed statistically significant strong negative relationships, those r-values of $\mathrm{pH}$ and $\mathrm{K}$ showed statistically significant moderate positive relationships, and those r-values of Na, BLG, amylase, lipase AST, ALT, BLU, and CRE depicted statistically significant moderate negative relationships. 
Table (1): Distribution pattern and statistical differences between genders and age among HC-group, AntiChE-group, and Intoxicated-groups.

\begin{tabular}{|l|l|l|l|l|l|}
\hline \multirow{2}{*}{ Variables } & \multirow{2}{*}{ HC-group } & \multirow{2}{*}{ AntiChE } & \multicolumn{3}{c|}{ Intoxicated-groups } \\
\cline { 4 - 6 } & & & Mild & Moderate & Severe \\
\hline Gender & & & & \\
\hline Male $^{@}$ & $9(36)$ & $30(40)$ & $17(22.67)$ & $8(10.67)$ & $5(6.67)$ \\
\hline Female $^{@}$ & $16(64)$ & $45(60)$ & $21(28.00)$ & $14(18.67)$ & $10(13.33)$ \\
\hline Sex ratio & $1: 1.77$ & $1: 1.5$ & $1: 1.24$ & $1: 1.75$ & $1: 2$ \\
\hline$X^{2}$ score & \multicolumn{5}{|c|}{0.902} \\
\hline P-value & \multicolumn{5}{|c|}{$0.924^{\mathrm{NS}}$} \\
\hline Age (Year) & \multicolumn{5}{|c|}{0.8291} \\
\hline Mean \pm SEM & $25.16 \pm 1.37$ & $23.82 \pm 0.82$ & $22.73 \pm 1.16$ & $24.15 \pm 1.52$ & $26.1 \pm 1.75$ \\
\hline F-statistic ${ }^{\#}$ & \multicolumn{5}{|c|}{$0.5082^{\mathrm{NS}}$} \\
\hline P-value & \multicolumn{5}{|c|}{} \\
\hline
\end{tabular}

$\mathrm{HC}=$ Healthy control; AntiChE=Anticholinesterase; $@=$ Number and percentage;

$\mathrm{X}^{2}=$ Chi-square; \#=F-test result of one-way analysis of variance (ANOVA);

$P=$ Probability; NS=Non-significant difference. $P$-value of $>0.05$ was considered nonsignificant.

Table (2): Distribution pattern of the AntiChE causative compounds according to their different subtypes.

\begin{tabular}{|c|c|c|c|c|c|}
\hline Compounds & Subtypes & $\begin{array}{c}\text { Number } \\
(\%)\end{array}$ & $\begin{array}{c}\text { Total } \\
(\%)\end{array}$ & $\begin{array}{c}\mathbf{X}^{2} \\
\text { score }\end{array}$ & P-value \\
\hline \multirow{4}{*}{ OPC } & Malathion (Carbophos) & $32(42.67)$ & \multirow{4}{*}{$\begin{array}{c}53 \\
(70.67)\end{array}$} & \multirow{7}{*}{2.38} & \multirow{7}{*}{$0.497^{\mathrm{NS}}$} \\
\hline & Dimethoate (Rogor) & $9(12.00)$ & & & \\
\hline & Diazinon (Basudin) & $7(9.33)$ & & & \\
\hline & Chloropyrifos (Lorsban) & $5(6.67)$ & & & \\
\hline \multirow{3}{*}{ CMC } & Aldicarb (Temik) & $14(18.67)$ & \multirow{3}{*}{$\begin{array}{c}22 \\
(29.33)\end{array}$} & & \\
\hline & Methomyl (Lannate) & $5(6.67)$ & & & \\
\hline & Carbofuran (Furadan) & $3(4.00)$ & & & \\
\hline
\end{tabular}

AntiChE=Anticholinesterase; $\mathrm{OPC}=$ Organophosphate compounds; $\mathrm{CMC}=\mathrm{Carbamate}$ compounds; $\%=$ Percentage; $\mathrm{X}^{2}=$ Chi-square; $\mathrm{P}=$ Probability; NS=Non-significant difference. $P$-value of $>0.05$ was considered non-significant. 
Table (3): Distribution pattern and chi-square statistical differences between the three Intoxicated-groups concerning the PSS, DHA, vital signs, GCS, and LHS at the time of admission.

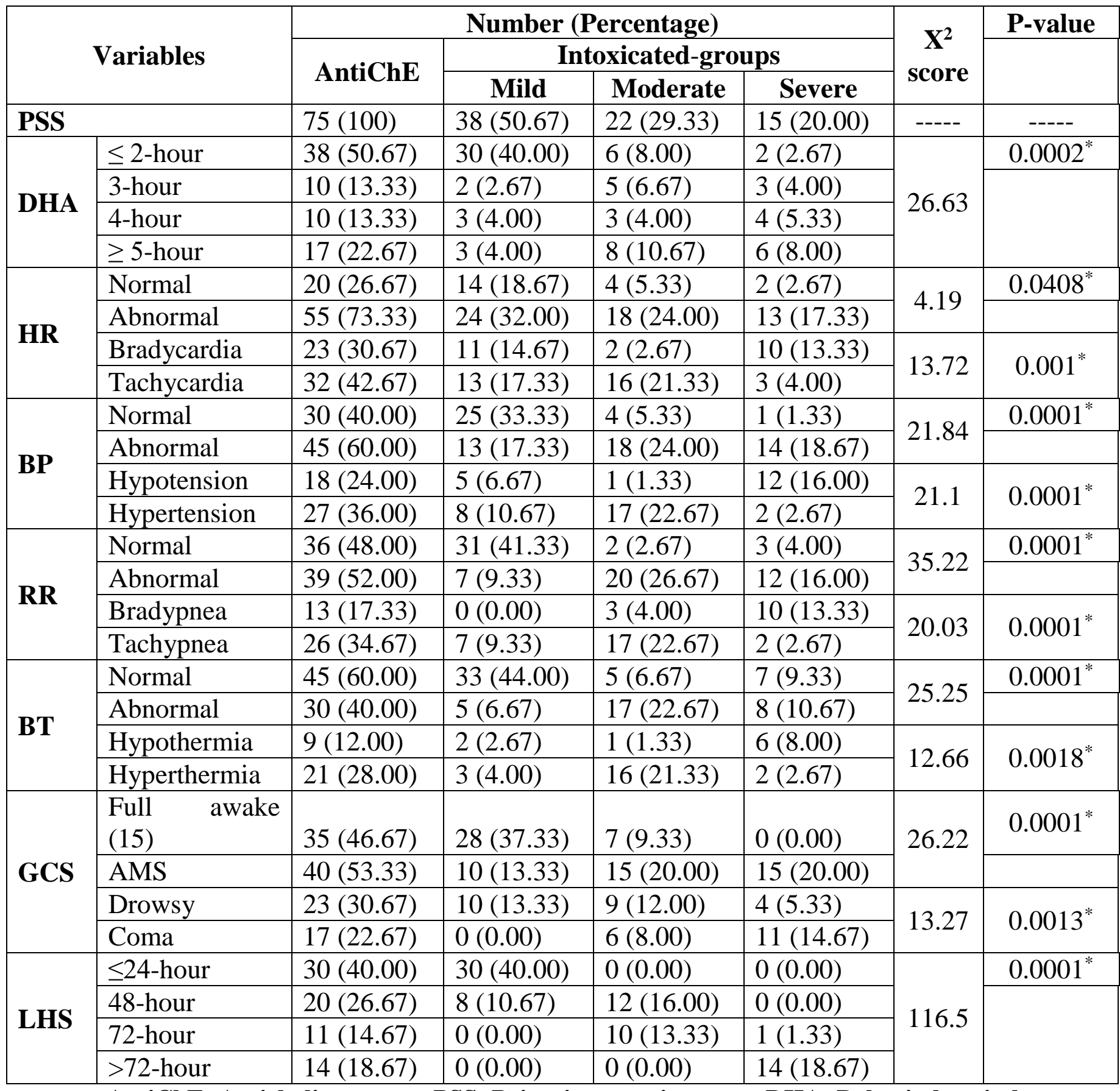

AntiChE=Anticholinesterase; PSS=Poisoning severity score; DHA=Delay in hospital arrival (hours); HR=Heart rate (beats/minute); DBP=Diastolic blood pressure (mmHg); $\mathrm{SBP}=$ Systolic blood pressure $(\mathrm{mmHg}) ; \mathrm{RR}=\mathrm{Respiratory}$ rate (breaths/minute); BT=Body temperature $\left({ }^{\circ} \mathrm{C}\right) ; \mathbf{G C S}=$ Glasgow Coma Scale; AMS=Altered mental status; LHS=Length of hospital stay (hours); $\mathrm{X}^{2}=$ Chi-square; $\mathrm{P}=$ Probability; *=Significant difference. $P$-value of $<0.05$ was considered significant 
Table (4): One-way analysis of variance (ANOVA) statistical differences between the Intoxicated-groups concerning the clinical findings at the time of admission.

\begin{tabular}{|c|c|c|c|c|c|c|}
\hline \multirow[t]{2}{*}{ 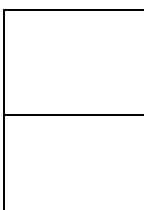 } & \multirow[t]{2}{*}{ Variables } & \multirow{2}{*}{$\begin{array}{l}\text { AntiChE- } \\
\text { group }\end{array}$} & \multicolumn{4}{|c|}{ Intoxicated-groups } \\
\hline & & & Mild & Moderate & Severe & $\begin{array}{l}\text { Severe vs } \\
\text { Moderate }\end{array}$ \\
\hline \multirow{2}{*}{$\begin{array}{l}\text { PSS } \\
\text { grade }\end{array}$} & $\begin{array}{l}\text { Mean } \quad \pm \\
\text { SEM }\end{array}$ & $1.69 \pm 0.09$ & $1 \pm 0.00$ & $2 \pm 0.00$ & $3 \pm 0.00$ & \\
\hline & P-value & $\begin{array}{l}---- \\
\end{array}$ & $\begin{array}{ll}---- \\
--\end{array}$ & $0.0001^{*}(\uparrow)$ & $0.0001^{*}(\uparrow)$ & $0.0001^{*}(\uparrow)$ \\
\hline \multirow[t]{2}{*}{ DHA } & $\begin{array}{l}\text { Mean } \quad \pm \\
\text { SEM }\end{array}$ & $3.5 \pm 0.32$ & $1.71 \pm 0.13$ & $4.14 \pm 0.33$ & $7.1 \pm 0.91$ & \\
\hline & P-value & $\begin{array}{ll}---- \\
\end{array}$ & $\begin{array}{ll}---- \\
\end{array}$ & $0.001^{*}(\uparrow)$ & $0.0001^{*}(\uparrow)$ & $0.002^{*}(\uparrow)$ \\
\hline \multirow[t]{2}{*}{ HR } & $\begin{array}{l}\text { Mean } \quad \pm \\
\text { SEM }\end{array}$ & $87.32 \pm 3.77$ & $82 \pm 3.38$ & $116.6 \pm 6.9$ & $57.87 \pm 6.1$ & \\
\hline & P-value & $\begin{array}{ll}----- \\
\end{array}$ & $\begin{array}{ll}----- \\
\end{array}$ & $0.0001^{*}(\uparrow)$ & $0.007^{*}(\downarrow)$ & $0.0001^{*}(\downarrow)$ \\
\hline \multirow[t]{2}{*}{ DBP } & $\begin{array}{l}\text { Mean } \quad \pm \\
\text { SEM }\end{array}$ & $74.91 \pm 2.57$ & $71.53 \pm 2.7$ & $94.1 \pm 3.94$ & $55.33 \pm 4.79$ & ----- \\
\hline & P-value & ----- & $\begin{array}{ll}---- \\
\end{array}$ & $0.0001^{*}(\uparrow)$ & $0.010^{*}(\downarrow)$ & $0.0001^{*}(\downarrow)$ \\
\hline \multirow[t]{2}{*}{ SBP } & $\begin{array}{l}\text { Mean } \quad \pm \\
\text { SEM }\end{array}$ & $118.69 \pm 3.83$ & $116.76 \pm 3.12$ & $147.95 \pm 5.98$ & $80.67 \pm 7.02$ & ----- \\
\hline & P-value & ----- & ----- & $0.0001^{*}(\uparrow)$ & $0.0001^{*}(\downarrow)$ & $0.0001^{*}(\downarrow)$ \\
\hline \multirow[t]{2}{*}{$\mathbf{R R}$} & $\begin{array}{l}\text { Mean } \quad \pm \\
\text { SEM }\end{array}$ & $23.41 \pm 1.1$ & $23.71 \pm 0.82$ & $30.32 \pm 2.1$ & $12.53 \pm 1.46$ & ----- \\
\hline & P-value & ----- & ----- & $0.002^{*}(\uparrow)$ & $0.0001^{*}(\downarrow)$ & $0.0001^{*}(\downarrow)$ \\
\hline \multirow[t]{2}{*}{ BT } & $\begin{array}{l}\text { Mean } \quad \pm \\
\text { SEM }\end{array}$ & $37.2 \pm 0.08$ & $37.06 \pm 0.3$ & $37.86 \pm 0.16$ & $36.55 \pm 0.15$ & ----- \\
\hline & P-value & ----- & ----- & $0.0001^{*}(\uparrow)$ & $0.003^{*}(\downarrow)$ & $0.0001^{*}(\downarrow)$ \\
\hline \multirow[t]{2}{*}{ GCS } & $\begin{array}{l}\text { Mean } \quad \pm \\
\text { SEM }\end{array}$ & $13.1 \pm 0.25$ & $14.55 \pm 0.14$ & $12.68 \pm 0.38$ & $9.93 \pm 0.34$ & \\
\hline & P-value & ----- & ----- & $0.0001^{*}(\downarrow)$ & $0.0001^{*}(\downarrow)$ & $0.0001^{*}(\downarrow)$ \\
\hline \multirow[t]{2}{*}{ LHS } & $\begin{array}{l}\text { Mean } \quad \pm \\
\text { SEM }\end{array}$ & $46.97 \pm 4.1$ & $20.76 \pm 1.16$ & $51.68 \pm 3.33$ & $106.5 \pm 5.62$ & \\
\hline & P-value & ----- & ----- & $0.0001^{*}(\uparrow)$ & $0.0001^{*}(\uparrow)$ & $0.0001^{*}(\uparrow)$ \\
\hline
\end{tabular}

AntiChE=Anticholinesterase; PSS=Poisoning severity score; DHA=Delay in hospital arrival (hours); HR=Heart rate (beats/minute); DBP=Diastolic blood pressure (mmHg);

SBP=Systolic blood pressure $(\mathrm{mmHg}) ; \mathrm{RR}=$ Respiratory rate (breaths/minute);

BT=Body temperature $\left({ }^{\circ} \mathrm{C}\right)$; GCS=Glasgow Coma Scale; LHS=Length of hospital stay (hours); SEM=Standard error of measurement; $P=$ Probability; *=Significant difference; $\uparrow=$ Increase; $\downarrow=$ Decrease. $P$-value of $<0.05$ was considered significant. 
Table (5): One-way analysis of variance (ANOVA) statistical comparison between HC-group and AntiChE-group at admission and discharge regarding to the mean values of the total and abnormal clinical findings.

\begin{tabular}{|c|c|c|c|c|c|}
\hline \multirow{2}{*}{\multicolumn{2}{|c|}{ Variables }} & \multirow{2}{*}{ HC-group } & \multicolumn{3}{|c|}{ AntiChE-group } \\
\hline & & & \multirow{2}{*}{$\begin{array}{c}\text { Admission } \\
1.69 \pm 0.09\end{array}$} & \multirow{2}{*}{$\begin{array}{l}\text { Discharge } \\
0 \pm 0.00\end{array}$} & \multirow{2}{*}{$\begin{array}{c}\text { Discharge vs Admissior } \\
----\end{array}$} \\
\hline \multirow{2}{*}{ PSS grades } & Mean \pm SEM & $0 \pm 0.00$ & & & \\
\hline & P-value & ----- & $0.0001^{*}$ & ----- & $0.0001^{*}(\downarrow)$ \\
\hline \multirow[b]{2}{*}{ Total HR } & Mean \pm SEM & $72.24 \pm 0.85$ & $87.32 \pm 3.77$ & $73.12 \pm 0.65$ & ----- \\
\hline & P-value & ----- & $0.009^{*}(\uparrow)$ & $0.89^{\mathrm{NS}}$ & $0.0001^{*}(\downarrow)$ \\
\hline \multirow{2}{*}{ Slow HR } & Mean \pm SEM & ----- & $49.13 \pm 1.1$ & $70.22 \pm 1$ & ----- \\
\hline & P-value & ----- & $0.0001^{*}(\downarrow)$ & $0.33^{\mathrm{NS}}$ & $0.0001^{*}(\uparrow)$ \\
\hline \multirow{2}{*}{ Rapid HR } & Mean \pm SEM & ----- & $119 \pm 3.21$ & $75.5 \pm 0.46$ & $\begin{array}{l}---- \\
\end{array}$ \\
\hline & P-value & ---- & $0.0001^{*}(\uparrow)$ & $0.60^{\mathrm{NS}}$ & $0.0001^{*}(\downarrow)$ \\
\hline \multirow{2}{*}{ Total DBP } & Mean \pm SEM & $75.88 \pm 0.87$ & $74.91 \pm 2.57$ & $70.24 \pm 0.58$ & ---- \\
\hline & P-value & ----- & $0.0 .89^{\mathrm{NS}}$ & $0.0 .31^{\mathrm{NS}}$ & $0.0 .18^{\mathrm{NS}}$ \\
\hline \multirow{2}{*}{ Low DBP } & Mean \pm SEM & ---- & $50.17 \pm 1.1$ & $73.16 \pm 1.1$ & ----- \\
\hline & P-value & ----- & $0.0001^{*}(\downarrow)$ & $0.10^{\mathrm{NS}}$ & $0.0001^{*}(\uparrow)$ \\
\hline \multirow{2}{*}{ High DBP } & Mean \pm SEM & ----- & $102.78 \pm 0.82$ & $74.11 \pm 0.83$ & $-\cdots$ \\
\hline & P-value & ----- & $0.0001^{*}(\uparrow)$ & $0.33^{\mathrm{NS}}$ & $0.0001^{*}(\downarrow)$ \\
\hline \multirow{2}{*}{ Total SBP } & Mean \pm SEM & $115.2 \pm 0.87$ & $118.69 \pm 3.83$ & $108.97 \pm 0.72$ & ---- \\
\hline & P-value & ----- & $0.88^{\mathrm{NS}}$ & $0.56^{\mathrm{NS}}$ & $0.024^{*}(\downarrow)$ \\
\hline \multirow{2}{*}{ Low SBP } & Mean \pm SEM & ----- & $73.78 \pm 2.26$ & $110.89 \pm 1.39$ & ---- \\
\hline & P-value & ----- & $0.0001^{*}(\downarrow)$ & $0.072^{\mathrm{NS}}$ & $0.0001^{*}(\uparrow)$ \\
\hline \multirow{2}{*}{ High SBP } & Mean \pm SEM & ----- & $156.44 \pm 1.77$ & $112.93 \pm 1.03$ & \\
\hline & P-value & ----- & $0.0001^{*}(\uparrow)$ & $0.49^{\mathrm{NS}}$ & $0.0001^{*}(\downarrow)$ \\
\hline \multirow{2}{*}{ Total RR } & Mean \pm SEM & $19.44 \pm 0.46$ & $23.41 \pm 1.1$ & $19.84 \pm 0.25$ & ----- \\
\hline & P-value & ---- & $0.017^{*}(\uparrow)$ & $0.958^{\mathrm{NS}}$ & $0.002^{*}(\downarrow)$ \\
\hline \multirow[b]{2}{*}{ Slow RR } & Mean \pm SEM & ----- & $10.54 \pm 0.18$ & $19.31 \pm 0.35$ & ----- \\
\hline & P-value & ----- & $0.0001^{*}(\downarrow)$ & $0.89^{\mathrm{NS}}$ & $0.0001^{*}(\uparrow)$ \\
\hline \multirow{2}{*}{ Rapid RR } & Mean \pm SEM & ----- & $29.77 \pm 0.52$ & $18.04 \pm 0.33$ & ----- \\
\hline & P-value & ----- & $0.0001^{*}(\uparrow)$ & $0.055^{\mathrm{NS}}$ & $0.0001^{*}(\downarrow)$ \\
\hline \multirow{2}{*}{ Total BT } & Mean \pm SEM & $37.07 \pm 0.01$ & $37.2 \pm 0.08$ & $37.04 \pm 0.01$ & ----- \\
\hline & P-value & ----- & $0.57^{\mathrm{NS}}$ & $0.89^{\mathrm{NS}}$ & $0.10^{\mathrm{NS}}$ \\
\hline \multirow{2}{*}{ Low BT } & Mean \pm SEM & ---- & $36.17 \pm 0.13$ & $37.1 \pm 0.03$ & ----- \\
\hline & P-value & ----- & $0.0001^{*}(\downarrow)$ & $0.90^{\mathrm{NS}}$ & $0.0001^{*}(\uparrow)$ \\
\hline \multirow{2}{*}{ High BT } & Mean \pm SEM & ----- & $38 \pm 0.14$ & $37.08 \pm 0.02$ & \\
\hline & P-value & ----- & $0.0001^{*}(\uparrow)$ & $0.90^{\mathrm{NS}}$ & $0.0001^{*}(\downarrow)$ \\
\hline \multirow{2}{*}{ Total GCS } & Mean \pm SEM & $15 \pm 0.00$ & $13.08 \pm 0.25$ & $14.63 \pm 0.06$ & ----- \\
\hline & P-value & ----- & $0.0001^{*}(\downarrow)$ & $0.63^{\mathrm{NS}}$ & $0.0001^{*}(\uparrow)$ \\
\hline \multirow{2}{*}{$\begin{array}{l}\text { Altered } \\
\text { GCS }\end{array}$} & Mean \pm SEM & ----- & $11.4 \pm 0.27$ & $14.38 \pm 0.08$ & ----- \\
\hline & P-value & ----- & $0.0001^{*}(\downarrow)$ & $0.065^{\mathrm{NS}}$ & $0.0001^{*}(\uparrow)$ \\
\hline
\end{tabular}

$\mathrm{HC}=$ Healthy control; AntiChE=Anticholinesterase; $\mathrm{PSS}=$ Poisoning severity score; $\mathrm{HR}=\mathrm{Heart}$ rate (beats/minute); DBP=Diastolic blood pressure $(\mathrm{mmHg})$; SBP=Systolic blood pressure $(\mathrm{mmHg}) ; \mathrm{RR}=$ Respiratory rate $\left(\right.$ breaths/minute); BT=Body temperature $\left({ }^{\circ} \mathrm{C}\right)$; GCS=Glasgow

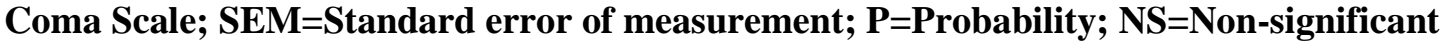
difference; *=Significant difference; $\uparrow=$ Increase; $\downarrow=$ Decrease. $P$-value of $>0.05$ was considered non-significant; $P$-value of $<0.05$ was considered significant. 
Table (6): Pearson's correlation analysis between PSS, GCS, and BuChE with the clinical findings of AntiChE-group at the time of admission.

\begin{tabular}{|l|c|c|r|r|r|r|}
\hline \multirow{2}{*}{ Variables } & \multicolumn{2}{|c|}{ PSS } & \multicolumn{2}{c|}{ GCS } & \multicolumn{2}{c|}{ BuChE } \\
\cline { 2 - 7 } & r-value & P-value & r-value & P-value & r-value & P-value \\
\hline DHA & $0.8595^{\$}$ & $0.0001^{*}$ & $-0.8839^{\$}$ & $0.0001^{*}$ & $-0.8037^{\$}$ & $0.0001^{*}$ \\
\hline Total HR & $-0.7729^{\$}$ & $0.0001^{*}$ & $0.5311^{\&}$ & $0.0001^{*}$ & $0.7848^{\$}$ & $0.0001^{*}$ \\
\hline Slow HR & $-0.8902^{\$}$ & $0.0001^{*}$ & $0.9482^{\$}$ & $0.0001^{*}$ & $0.6081^{\&}$ & $0.0035^{*}$ \\
\hline Rapid HR & $-0.5771^{\&}$ & $0.0007^{*}$ & $0.7887^{\$}$ & $0.0001^{*}$ & $0.5397^{\&}$ & $0.0018^{*}$ \\
\hline Total DBP & $-0.8385^{\$}$ & $0.0001^{*}$ & $0.6666^{\&}$ & $0.0001^{*}$ & $0.6274^{\&}$ & $0.0001^{*}$ \\
\hline Low DBP & $-0.8511^{\$}$ & $0.0001^{*}$ & $0.8254^{\$}$ & $0.0001^{*}$ & $0.527^{\&}$ & $0.0246^{*}$ \\
\hline High DBP & $-0.7915^{\$}$ & $0.0001^{*}$ & $0.5692^{\&}$ & $0.0019^{*}$ & $0.6277^{\&}$ & $0.0005^{*}$ \\
\hline Total SBP & $-0.7227^{\&}$ & $0.0001^{*}$ & $0.5703^{\&}$ & $0.0001^{*}$ & $0.6396^{\&}$ & $0.0001^{*}$ \\
\hline Low SBP & $-0.7651^{\$}$ & $0.0002^{*}$ & $0.843^{\$}$ & $0.0001^{*}$ & $0.664^{\&}$ & $0.0027^{*}$ \\
\hline High SBP & $-0.7693^{\$}$ & $0.0001^{*}$ & $0.5417^{\&}$ & $0.0035^{*}$ & $0.5944^{\&}$ & $0.0011^{*}$ \\
\hline Total RR & $-0.5584^{\&}$ & $0.0001^{*}$ & $0.5302^{\&}$ & $0.0001^{*}$ & $0.7611^{\$}$ & $0.0001^{*}$ \\
\hline Slow RR & $-0.5752^{\&}$ & $0.0397^{*}$ & $0.8564^{\$}$ & $0.0001^{*}$ & $0.7793^{\$}$ & $0.0017^{*}$ \\
\hline Rapid RR & $-0.6825^{\&}$ & $0.0001^{*}$ & $0.7862^{\$}$ & $0.0001^{*}$ & $0.7459^{\&}$ & $0.0001^{*}$ \\
\hline Total BT & $-0.7035^{\&}$ & $0.0001^{*}$ & $0.7239^{\&}$ & $0.0001^{*}$ & $0.6083^{\&}$ & $0.0001^{*}$ \\
\hline Low BT & $-0.7501^{\$}$ & $0.0199^{*}$ & $0.8355^{\$}$ & $0.005^{*}$ & $0.6841^{\&}$ & $0.0421^{*}$ \\
\hline High BT & $-0.6592^{\&}$ & $0.0012^{*}$ & $0.7231^{\&}$ & $0.0002^{*}$ & $0.5132^{\&}$ & $0.0173^{*}$ \\
\hline PSS & ----- & ----- & $-0.8094^{\$}$ & $0.0001^{*}$ & $-0.9173^{\$}$ & $0.0001^{*}$ \\
\hline GCS & ----- & ----- & ----- & ----- & $0.8883^{\$}$ & $0.0001^{*}$ \\
\hline LHS & $0.912^{\$}$ & $0.0001^{*}$ & $-0.7751^{\$}$ & $0.0001^{*}$ & $-0.9433^{\$}$ & $0.0001^{*}$ \\
\hline
\end{tabular}

PSS=Poisoning severity score; GCS=Glasgow Coma Scale;

BuChE=Butyrylcholinesterase; AntiChE=Anticholinesterase; DHA=Delay in hospital arrival (hours); HR=Heart rate (beats/minute); DBP=Diastolic blood pressure (mmHg);

$\mathrm{SBP}=$ Systolic blood pressure $(\mathrm{mmHg}) ; \mathrm{RR}=\mathrm{Respiratory}$ rate (breaths/minute);

BT=Body temperature $\left({ }^{\circ} \mathrm{C}\right)$; LHS=Length of hospital stay (hours); *=Significant difference. P-value of $<0.05$ was considered significant. \$=Strong correlation; $\&=$ Moderate correlation. 
Table (7): The distribution patterns and chi-square statistical differences between the intoxicated study groups concerning the normal and abnormal laboratory findings of the investigated biochemical and enzymatic biomarkers at the time of admission.

\begin{tabular}{|c|c|c|c|c|c|c|c|}
\hline \multirow{3}{*}{\multicolumn{2}{|c|}{ Variables }} & \multirow{2}{*}{\multicolumn{4}{|c|}{$\begin{array}{c}\text { Number (Percentage) } \\
\text { Grouns }\end{array}$}} & \multirow{3}{*}{$\begin{array}{c}\mathbf{X}^{2} \\
\text { score }\end{array}$} & \multirow{3}{*}{ P-value } \\
\hline & & & Gr & ups & & & \\
\hline & & \multirow{2}{*}{$\frac{\text { AntiChE }}{45(60)}$} & \multirow{2}{*}{$\begin{array}{c}\text { Mild } \\
33(44)\end{array}$} & \multirow{2}{*}{$\begin{array}{l}\text { Moderate } \\
6(8)\end{array}$} & \multirow{2}{*}{$\begin{array}{l}\text { Severe } \\
6(8)\end{array}$} & & \\
\hline \multirow{2}{*}{ pH } & Normal & & & & & \multirow{2}{*}{23.73} & \multirow{2}{*}{$0.0001^{*}$} \\
\hline & Decreased & $30(40)$ & $5(6.67)$ & $16(21.33)$ & $9(12)$ & & \\
\hline \multirow{2}{*}{$\mathbf{N a}$} & \begin{tabular}{|l|} 
Normal \\
\end{tabular} & $54(72)$ & $36(48)$ & $14(18.67)$ & $4(5.33)$ & \multirow{2}{*}{25.8} & \multirow{2}{*}{$0.0001^{*}$} \\
\hline & \begin{tabular}{|l|} 
Increased \\
\end{tabular} & $21(28)$ & $2(2.67)$ & $8(10.67)$ & 11 (14.67) & & \\
\hline \multirow[b]{2}{*}{$\mathbf{K}$} & Normal & $33(44)$ & $30(40)$ & $2(2.67)$ & $1(1.33)$ & \multirow[b]{2}{*}{38.2} & \multirow{2}{*}{0.0001} \\
\hline & Decreased & $42(56)$ & 8 (10.67) & $20(26.67)$ & $14(18.67)$ & & \\
\hline \multirow{3}{*}{ BLG } & Normal & $25(33.33)$ & $22(29.33)$ & $2(2.67)$ & $1(1.33)$ & \multirow{3}{*}{23.1} & \multirow{3}{*}{$0.0001^{\prime}$} \\
\hline & \begin{tabular}{|l|} 
Increased \\
\end{tabular} & $43(57.33)$ & $12(16.00)$ & $18(24.00)$ & $13(17.33)$ & & \\
\hline & Decreased & $7(9.33)$ & $4(5.33)$ & $2(2.67)$ & $1(1.33)$ & & \\
\hline \multirow{4}{*}{ BuChE } & $50 \%$ & $32(42.67)$ & $32(42.67)$ & $0(0.00)$ & $0(0.00)$ & \multirow{4}{*}{111.36} & \multirow{4}{*}{0.0001} \\
\hline & $20-50 \%$ & $24(32.00)$ & $6(8.00)$ & $18(24.00)$ & $0(0.00)$ & & \\
\hline & $10-20 \%$ & $7(9.33)$ & $0(0.00)$ & $4(5.33)$ & $3(4.00)$ & & \\
\hline & $<10 \%$ & $12(16.00)$ & $0(0.00)$ & $0(0.00)$ & $12(16.00)$ & & \\
\hline \multirow{2}{*}{ CK-MB } & Normal & $47(82.46)$ & $38(66.67)$ & $7(12.28)$ & $2(3.51)$ & \multirow{2}{*}{47.19} & \multirow{2}{*}{$0.0001^{*}$} \\
\hline & Increased & $28(49.12)$ & $0(0.00)$ & $15(26.32)$ & $13(22.81)$ & & \\
\hline \multirow{2}{*}{ cTnI } & Normal & $57(76.00)$ & $38(50.67)$ & $14(18.67)$ & $5(6.67)$ & \multirow{2}{*}{28.81} & \multirow{2}{*}{$0.0001^{*}$} \\
\hline & Increased & $18(24.00)$ & $0(0.00)$ & $8(10.67)$ & $10(13.33)$ & & \\
\hline A mylaco & Normal & $50(66.67)$ & $33(44.00)$ & $11(14.67)$ & $6(8.00)$ & & \\
\hline Amyiase & Increased & $25(33.33)$ & $5(6.67)$ & $11(14.67)$ & $9(12.00)$ & & \\
\hline Lin & Normal & $55(73.33)$ & $36(48.00)$ & $12(16.00)$ & $7(9.33)$ & & \\
\hline & Increased & $20(26.67)$ & $2(2.67)$ & $10(13.33)$ & $8(10.67)$ & & \\
\hline AST & Normal & $34(45.33)$ & $32(42.67)$ & $1(1.33)$ & $1(1.33)$ & 4699 & $00001^{*}$ \\
\hline ASI & Increased & $41(54.67)$ & $6(8.00)$ & $21(28.00)$ & 14 (18.67) & & \\
\hline ALT & Normal & $42(56.00)$ & $35(46.67)$ & $4(5.33)$ & $3(4.00)$ & 40.76 & $0.0001^{*}$ \\
\hline ALI & Increased & $33(44.00)$ & $3(4.00)$ & $18(24.00)$ & $12(16.00)$ & 40.10 & 0.0001 \\
\hline BLI & Normal & $42(56.00)$ & $35(46.67)$ & $5(6.67)$ & $2(2.67)$ & & 0000 \\
\hline DLU & Increased & $33(44.00)$ & $3(4.00)$ & $17(22.67)$ & $13(17.33)$ & & \\
\hline $\mathbf{C}$ & Normal & $46(61.33)$ & $34(45.33)$ & $8(10.67)$ & $4(5.33)$ & & 0.06 \\
\hline CNE & Increased & $29(38.67)$ & $4(5.33)$ & 14 (18.67) & $11(14.67)$ & & \\
\hline
\end{tabular}

AntiChE=Anticholinesterase; Na=Sodium; K=Potassium; BLG=Blood glucose;

BuChE=Butyrylcholinesterase; CK-MB=Creatine kinase-myocardial band;

cTnI=Cardiac troponin I; AST=Aspartate aminotransferase; ALT=Alanine

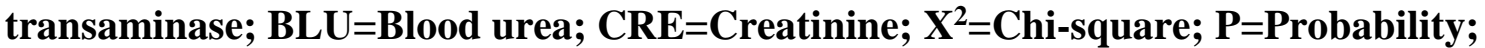
$*=$ Significant difference. P-value of $<0.05$ was considered significant. 
Table (8): One-way analysis of variance (ANOVA) statistical differences between the Intoxicated-groups concerning the mean values of the investigated biochemical and enzymatic biomarkers at the time of admission.

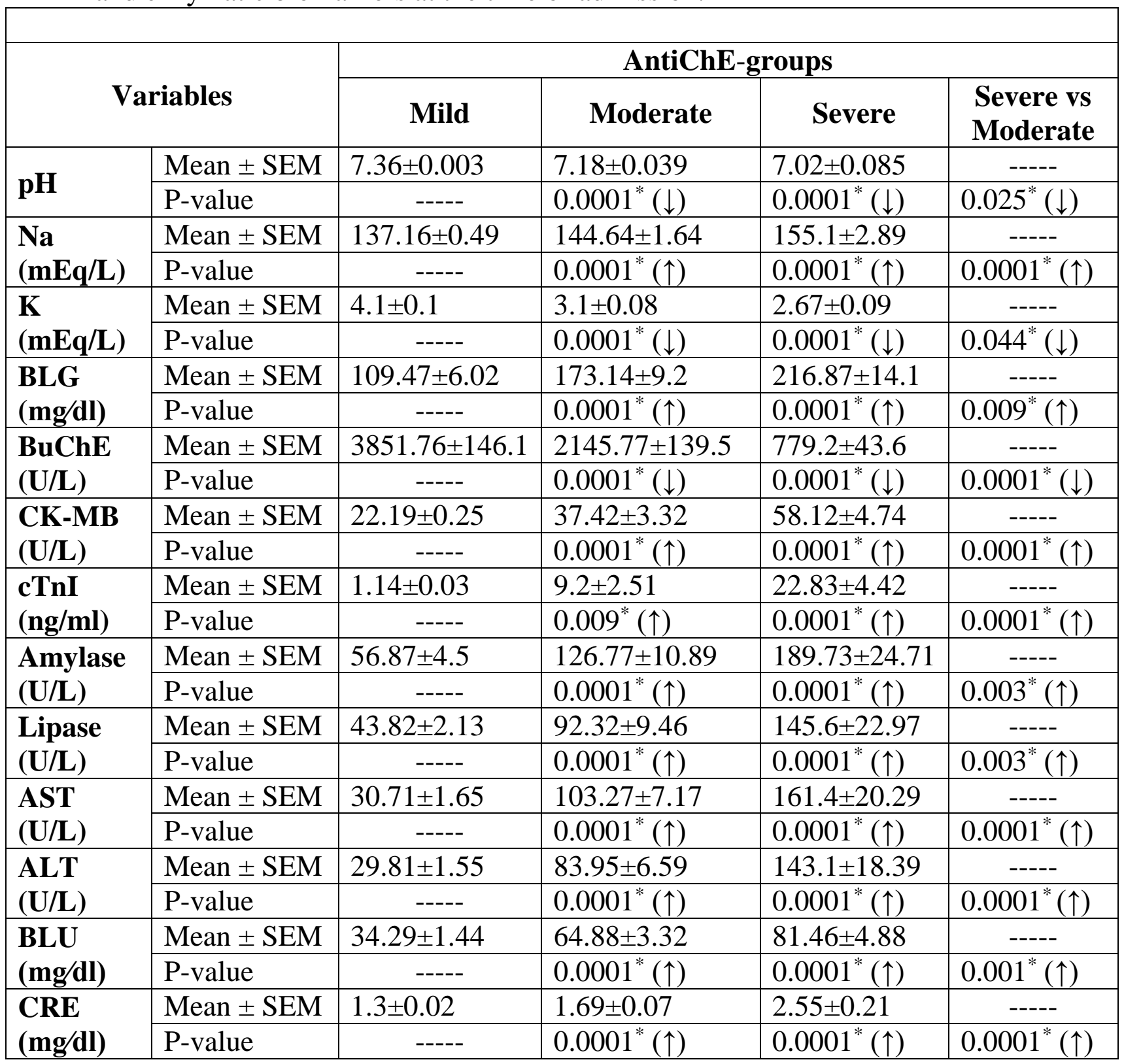

AntiChE=Anticholinesterase; $\mathrm{Na}=$ Sodium; K=Potassium; $\mathrm{BLG}=\mathrm{Blood}$ glucose;

BuChE=Butyrylcholinesterase; $\mathrm{CK}-\mathrm{MB}=$ Creatine kinase-myocardial band; cTnI=Cardiac troponin I; AST=Aspartate aminotransferase; ALT=Alanine transaminase; $B L U=B l o o d$ urea; $C R E=$ Creatinine; $S E M=S t a n d a r d$ error of measurement; $P=$ Probability; *=Significant difference; $\uparrow=$ Increase; $\downarrow=$ Decrease. $P$-value of $<0.05$ was considered significant. 
Table (9): One-way analysis of variance (ANOVA) statistical comparison between HC-group and AntiChE-groups at admission and discharge regarding to the mean values of the investigated biochemical and enzymatic biomarkers.

\begin{tabular}{|c|c|c|c|c|c|}
\hline & \multirow[b]{2}{*}{ HC-group } & \multicolumn{3}{|c|}{ AntiChE-groups } \\
\hline & & & \multirow{2}{*}{$\begin{array}{c}\text { Admission } \\
7.24 \pm 0.025\end{array}$} & \multirow{2}{*}{$\begin{array}{l}\text { Discharge } \\
7.36 \pm 0.002\end{array}$} & \multirow{2}{*}{$\begin{array}{c}\text { Discharge } \\
\text { vs } \\
\text { Admission } \\
\end{array}$} \\
\hline \multirow{2}{*}{ pH } & \pm SEM & $7.37 \pm 0$. & & & \\
\hline & P-value & ---- & $0.0001^{*}(\downarrow)$ & $0.944^{\mathrm{NS}}$ & $0.0001^{*}(\uparrow)$ \\
\hline \multirow{2}{*}{$\begin{array}{l}\mathrm{Na} \\
(\mathrm{mEq} / \mathrm{L})\end{array}$} & Mean \pm SEM & $138.12 \pm 0.59$ & $142.93 \pm 1.11$ & $137.51 \pm 0.29$ & ----- \\
\hline & P-value & ----- & $0.005^{*}(\uparrow)$ & $0.915^{\mathrm{NS}}$ & $0.0001^{*}(\downarrow)$ \\
\hline \multirow{2}{*}{$\begin{array}{l}\mathrm{K} \\
(\mathrm{mEq} / \mathrm{L})\end{array}$} & Mean \pm SEM & $4.31 \pm 0.12$ & $3.49 \pm 0.09$ & $4.04 \pm 0.05$ & ----- \\
\hline & P-value & $\begin{array}{ll}---- \\
\end{array}$ & $0.0001^{*}(\downarrow)$ & $0.15^{\mathrm{NS}}$ & $0.0001^{*}(\uparrow)$ \\
\hline \multirow{2}{*}{$\begin{array}{l}\text { BLG } \\
(\mathrm{mg} / \mathrm{dl})\end{array}$} & Mean \pm SEM & $113.36 \pm 3.77$ & $149.63 \pm 7$ & $107.19 \pm 1.98$ & $\begin{array}{ll}----- \\
\end{array}$ \\
\hline & P-value & ----- & $0.0001^{*}(\uparrow)$ & $0.78^{\mathrm{NS}}$ & $0.0001^{*}(\downarrow)$ \\
\hline \multirow{2}{*}{$\begin{array}{l}\text { BuChE } \\
(\mathrm{U} / \mathrm{L})\end{array}$} & Mean \pm SEM & $7403.7 \pm 324.7$ & $2736.8 \pm 165.4$ & $3975.3 \pm 170.4$ & ----- \\
\hline & P-value & ----- & $0.0001^{*}(\downarrow)$ & $0.0001^{*}(\downarrow)$ & $0.0001^{*}(\uparrow)$ \\
\hline \multirow{2}{*}{$\begin{array}{l}\text { CK-MB } \\
(\mathrm{U} / \mathrm{L})\end{array}$} & Mean \pm SEM & $19.31 \pm 0.48$ & $33.84 \pm 2.1$ & $21.32 \pm 0.28$ & ----- \\
\hline & P-value & $\begin{array}{ll}---- \\
\end{array}$ & $0.0001^{*}(\uparrow)$ & $0.73^{\mathrm{NS}}$ & $0.0001^{*}(\downarrow)$ \\
\hline \multirow{2}{*}{$\begin{array}{l}\text { cTnI } \\
(\mathrm{ng} / \mathrm{ml})\end{array}$} & Mean \pm SEM & $1.11 \pm 0.04$ & $7.84 \pm 1.48$ & $1.22 \pm 0.03$ & $\begin{array}{ll}----- \\
\end{array}$ \\
\hline & P-value & ----- & $0.002^{*}(\uparrow)$ & $0.89^{\mathrm{NS}}$ & $0.0001^{*}(\downarrow)$ \\
\hline \multirow{2}{*}{$\begin{array}{l}\text { Amylase } \\
(\mathrm{U} / \mathrm{L})\end{array}$} & Mean \pm SEM & $39.28 \pm 2.38$ & $100.16 \pm 9$ & $49.17 \pm 2$ & $\begin{array}{ll}---- \\
\end{array}$ \\
\hline & P-value & ----- & $0.0001^{*}(\uparrow)$ & $0.68^{\mathrm{NS}}$ & $0.0001^{*}(\downarrow)$ \\
\hline \multirow{2}{*}{$\begin{array}{l}\text { Lipase } \\
\text { (U/L) }\end{array}$} & Mean \pm SEM & $35.45 \pm 1.93$ & $78.4 \pm 7.1$ & $42.29 \pm 1.41$ & \\
\hline & P-value & ----- & $0.0001^{*}(\uparrow)$ & $0.77^{\mathrm{NS}}$ & $0.0001^{*}(\downarrow)$ \\
\hline \multirow{2}{*}{$\begin{array}{l}\text { AST } \\
(\mathbf{U} / \mathbf{L})\end{array}$} & Mean \pm SEM & $26.48 \pm 1.28$ & $78.13 \pm 7.56$ & $31.55 \pm 0.83$ & ----- \\
\hline & $\mathrm{P}$-value & ----- & $0.0001^{*}(\uparrow)$ & $0.85^{\mathrm{NS}}$ & $0.0001^{*}(\downarrow)$ \\
\hline \multirow{2}{*}{$\begin{array}{l}\mathbf{A L T} \\
(\mathbf{U} / \mathbf{L})\end{array}$} & Mean \pm SEM & $25.6 \pm 1.35$ & $68.35 \pm 6.58$ & $30.29 \pm 0.61$ & ----- \\
\hline & $\mathrm{P}$-value & ----- & $0.0001^{*}(\uparrow)$ & $0.84^{\mathrm{NS}}$ & $0.0001^{*}(\downarrow)$ \\
\hline \multirow{2}{*}{$\begin{array}{l}\text { BLU } \\
(\mathrm{mg} / \mathrm{dl})\end{array}$} & Mean \pm SEM & $29.53 \pm 1.15$ & $52.7 \pm 2.74$ & $33.2 \pm 0.72$ & ----- \\
\hline & P-value & ----- & $0.0001^{*}(\uparrow)$ & $0.58^{\mathrm{NS}}$ & $0.0001^{*}(\downarrow)$ \\
\hline \multirow{2}{*}{$\begin{array}{l}\text { CRE } \\
(\mathrm{mg} / \mathrm{dl})\end{array}$} & Mean \pm SEM & $1.11 \pm 0.05$ & $1.66 \pm 0.07$ & $1.27 \pm 0.01$ & ----- \\
\hline & P-value & ----- & $0.0001^{*}(\uparrow)$ & $0.21^{\mathrm{NS}}$ & $0.0001^{*}(\downarrow)$ \\
\hline
\end{tabular}

$\mathrm{HC}=$ Healthy control; AntiChE=Anticholinesterase; $\mathrm{Na}=$ Sodium; $\mathrm{K}=$ Potassium;

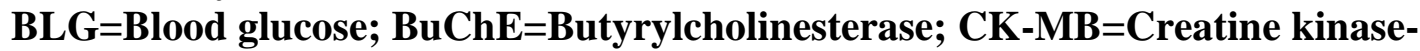
myocardial band; $\mathbf{c T n I}=$ Cardiac troponin I; AST=Aspartate aminotransferase; ALT=Alanine transaminase; $B L U=B l o o d$ urea; $C R E=C$ reatinine; $S E M=$ Standard error of measurement; $P=$ Probability; $N S=$ Non-significant difference; *=Significant difference; $\uparrow=$ Increase; $\downarrow=$ Decrease. $P$-value of $>0.05$ was considered non-significant; $P$ value of $<0.05$ was considered significant. 
Table (10): Pearson's correlation analysis between PSS, GCS, and BuChE with laboratory findings of the investigated biochemical and enzymatic biomarkers of AntiChE-groups at the time of admission.

\begin{tabular}{|l|l|l|l|l|l|l|}
\hline \multirow{2}{*}{ Parameters } & \multicolumn{2}{c|}{ PSS } & \multicolumn{2}{c|}{ GCS } & \multicolumn{2}{c|}{ BuChE } \\
\cline { 2 - 7 } & r-value & P-value & \multicolumn{1}{c|}{ r-value } & P-value & \multicolumn{1}{c|}{ r-value } & P-value \\
\hline $\mathrm{pH}$ & $-0.8438^{\$}$ & $0.0001^{*}$ & $0.5706^{\&}$ & $0.0001^{*}$ & $0.6316^{\&}$ & $0.0001^{*}$ \\
\hline $\mathrm{Na}(\mathrm{mEq} / \mathrm{L})$ & $0.7874^{\$}$ & $0.0001^{*}$ & $-0.5124^{\&}$ & $0.0001^{*}$ & $-0.6197^{\&}$ & $0.0001^{*}$ \\
\hline $\mathrm{K}(\mathrm{mEq} / \mathrm{L})$ & $-0.8533^{\$}$ & $0.0001^{*}$ & $0.5314^{\&}$ & $0.0001^{*}$ & $0.5354^{\&}$ & $0.0001^{*}$ \\
\hline BLG $(\mathrm{mg} / \mathrm{dl})$ & $0.9149^{\$}$ & $0.0001^{*}$ & $-0.6521^{\&}$ & $0.0001^{*}$ & $-0.5842^{\&}$ & $0.0001^{*}$ \\
\hline $\mathrm{CK}-\mathrm{MB}(\mathrm{U} / \mathrm{L})$ & $0.7897^{\$}$ & $0.0001^{*}$ & $-0.5136^{\&}$ & $0.0001^{*}$ & $-0.8264^{\$}$ & $0.0001^{*}$ \\
\hline cTnI (ng/ml) & $0.7931^{\$}$ & $0.0001^{*}$ & $-0.5959^{\&}$ & $0.0001^{*}$ & $-0.7991^{\$}$ & $0.0001^{*}$ \\
\hline Amylase (U/L) & $0.6583^{\&}$ & $0.0001^{*}$ & $-0.4314^{@}$ & $0.0001^{*}$ & $-0.6903^{\&}$ & $0.0001^{*}$ \\
\hline Lipase (U/L) & $0.5126^{\&}$ & $0.0001^{*}$ & $-0.3615^{\circledR}$ & $0.0014^{*}$ & $-0.6129^{\&}$ & $0.0001^{*}$ \\
\hline AST (U/L) & $0.7808^{\$}$ & $0.0001^{*}$ & $-0.6866^{\&}$ & $0.0001^{*}$ & $-0.7023^{\&}$ & $0.0001^{*}$ \\
\hline ALT (U/L) & $0.7733^{\$}$ & $0.0001^{*}$ & $-0.6493^{\&}$ & $0.0001^{*}$ & $-0.7495^{\&}$ & $0.0001^{*}$ \\
\hline BLU (mg/dl) & $0.6915^{\&}$ & $0.0001^{*}$ & $-0.5598^{\&}$ & $0.0001^{*}$ & $-0.6859^{\&}$ & $0.0001^{*}$ \\
\hline CRE (mg/dl) & $0.6291^{\&}$ & $0.0001^{*}$ & $-0.5929^{\&}$ & $0.0001^{*}$ & $-0.5334^{\&}$ & $0.0001^{*}$ \\
\hline
\end{tabular}

PSS=Poisoning severity score; GCS=Glasgow Coma Scale;

BuChE=Butyrylcholinesterase; AntiChE=Anticholinesterase; $\mathrm{Na}=$ Sodium;

$\mathrm{K}=$ Potassium; $\mathbf{B L G}=\mathrm{Blood}$ glucose; $\mathrm{CK}-\mathrm{MB}=$ Creatine kinase-myocardial band;

cTnI=Cardiac troponin I; AST=Aspartate aminotransferase; $\mathbf{A L T}=$ Alanine

transaminase; $\mathrm{BLU}=\mathrm{Blood}$ urea; $\mathrm{CRE}=$ Creatinine; $\mathrm{P}=$ Probability; $*=$ Significant difference. $P$-value of $<0.05$ was considered significant. $\$=$ Strong correlation; $\&=$ Moderate correlation; $@=$ Weak correlation.

\section{DISCUSSION}

Pesticide substances are extensively utilized in both developed and developing countries all over the world. These compounds have the potential to induce serious harmful effects on the health and death in human beings (Aslan et al., 2011).

The common use of AntiChE insecticides, especially the OPC and $\mathrm{CMC}$, for various purposes has been associated with intense hazards effects including severe acute and chronic poisonings in human and animal species (Kumar et al., 2010). Every year, millions of people worldwide are intentionally and unintentionally poisoned by pesticides, particularly OPC and CMC (Goldsmith et al., 2016).

In the current study, the number of intoxicated females was predominating over males with insignificant difference observed in the mean age between the study groups. These findings harmonize with those achieved by several authors who documenting that females outnumbered males (Exner and Ayala, 2009; Aslan et al., 2011). In contrast, other authors have reported predominance of AntiChE poisoning among males (Soltaninejad et al., 2007; Sam et al., 2009). The higher incidence of AntiChE poisoning among females may be due to the prevalence of the suicidal attempts by these products and different socio-economic problems.

The present study illustrated predominance of intoxication by OPC over CMC, which agrees with the results of other studies conducted in Egypt (Mansour and Gamalludin, 2008), Iran (Soltaninejad et al., 2007), and Turkey (Aslan et al., 2011). On the contrary, other researchers found more 
prevalence of poisoning by $\mathrm{CMC}$ than OPC (Saadeh et al., 1996). Uncontrolled sale and preferred use of the highly toxic OPC may be consider as the main reasons for this high number of poisoning by OPC (Sungur and Güven, 2001).

In this study, malathion and aldicarb were the most common causative agents involved in OPC and CMC poisoning, respectively. Similar to this finding, several authors have found predominant use of these agents in poisoning incidents among the intoxicated cases (Soltaninejad et al., 2007; Ibrahim et al., 2011). Contrariwise to this, other study revealed that dichlorvos and phenthoate OPC mostly involved in acute human poisoning (Saadeh et al., 1996; Abd El Al et al., 2016). The higher prevalence of these AntiChE subtypes may be attributed their wide availability, low mammalian toxicity, and global popularity in controlling arthropods, ectoparasites, human head and body lice, and household insects as well as they are very cheap, easily obtained, and sold in unregulated packets (AlAttar, 2010).

The present results emphasized predominance of patients with mild grade of PSS. Likewise, other studies have reported that most AntiChE poisoned patients presented to the hospital with mild intoxication as evaluated by PSS (WeissmannBrenner et al., 2002; Akdur et al., 2010). Paradoxical to this, Sam et al. (2009) and Azab (2015) have reported that the majority of AntiChE poisoned cases suffered from severe toxicity with higher PSS grades. The severity of poisoning by AntiChE compounds depends on the dose, the route of exposure, the type and pharmacokinetic properties of substance, and the previous health status of the affected individual (Soysal et al., 2011).

The current findings announced that the vast majority of intoxicated cases were admitted to the hospital within two-hour after toxic exposure, which is similar to the results of other articles (Kara et al., 2002; Karki et al., 2004; Vijayakumar et al., 2011). Contradiction to this, other studies have depicted that the minority of AntiChE poisoned cases presented to the hospital within two-hour or shown prolonged mean arrival time following toxic exposure (Ozer et al., 2007; Exner and Ayala, 2009). This discrepancy may be due to the fact that the greater numbers of patients came directly to the unit without transfers from another health care facility and their residency present nearby the hospital.

In this study, the duration of hospital stay has been found to be shorter than that reported in the other studies (Ozer et al., 2007; Kozacı et al., 2012). The length of hospital stay is directly proportional to the severity of poisoning, ingested dose, and arrival time to the hospital, which may contribute to this difference.

In the current study, various alternations in the vital signs and conscious level as evaluated by GCS were observed in some patients. Also, the mean values of vital signs and GCS scores of AntiChE-group on admission showed significant differences when compared with the corresponding values of both AntiChE-group at discharge and HC-group. However, these clinical changes were markedly improved at discharge as evidenced by insignificant differences from HCgroup.

Analogous to the current observations of alternations in heart rate, many previous studies showed 
more frequent occurrence of tachycardia than bradycardia among AntiChE intoxicated patients (Yurumez et al., 2007; Aslan et al., 2011; Vijayakumar et al., 2011). Tachycardia may occur via nicotinic effects on adrenal medulla, sympathetic ganglionic stimulation, hypoxia, and/or dehydration (Anand et al., 2009), while bradycardia results from the muscarinic effects of AntiChE compounds (Yurumez et al., 2007) or secondary to augmented vagal influence that shortens the atrial refractory period and increases the conduction time and the refractory period of sino-atrial and atrioventricular nodes (Vijayakumar et al, 2011).

Similar to the present results of abnormalities in blood pressure, preceding findings from many articles depicted more prevalent occurrence of hypertension than hypotension in AntiChE poisoned patients (Yurumez et al., 2007; Vijayakumar et al., 2011). In AntiChE poisoning, hypertension denotes nicotinic manifestation, whereas hypotension indicates cholinergic effect with vascular receptors overstimulation by circulating acetylcholine (Eddleston and Clark, 2011; Vijayakumar et al., 2011).

Poisoning by AntiChE compounds can induce either central respiratory depression with a decreased respiratory rate or tachypnea (Eddleston et al., 2006). Parallel with the current results of respiratory dysfunction, other investigators reported various respiratory complications following AntiChE poisoning with more manifestations of tachypnea than bradypnea (Bhattacharyya et al., 2011). On the contrary, the study of Yurumez et al. (2007) has shown that the majority of AntiChE poisoned cases experienced bradypnea. These complications may arise from acute stimulation of cholinergic receptors with subsequent development of bronchospasm and bronchoconstriction due to muscarinic effects, weakness and paralysis of respiratory diaphragm and intercostal muscles from nicotinic effects, and loss or cessation of central respiratory drive due to central effects, resulting in respiratory insufficiency, distress, dyspnea, failure, or apnea (Satoh, 2006).

Comparable with the current findings of changes in body temperature, several human reports have demonstrated considerable occurrence of hyperthermia than hypothermia after AntiChE poisoning. AntiChE compounds may interfere with the control of acetylcholine-regulated homeostasis, resulting in disturbance of normal thermoregulation mechanisms. Also, it has been suggested that changes in body temperature may be dose dependent. Generally, exposure to higher and lower doses of AntiChE compounds may be associated with hypothermia and hyperthermia, respectively (Yurumez et al., 2007; Talaie et al., 2012).

On the other hand, the results of several other publications regarding to AntiChE poisoning have illustrated either higher incidents of respiratory complications or less frequent occurrence of cardio-respiratory disturbances, tachycardia, tachypnea, and fall of blood pressure (Ozer et al., 2007; Titlić et al., 2008), which disagree with the present findings.

Acute AntiChE poisoning is one of the commonest causes of altered mental status. In the present study, clinical examination revealed that the main bulk of patients presented to the unit with different grades of altered mental 
status as assessed by GCS. This finding is in agreement with several other literatures which illustrating that the vast majority of AntiChE intoxicated patients had a low GCS scores (Sungur and Güven, 2001; Ozer et al., 2007; Kozacı et al., 2012). Unlikely, other studies showed a lower incidence of comatosed patients due to AntiChE poisoning (Baydin et al., 2007). The neurological disturbances and decrease in GCS score can be explained by several factors such as direct impact of AntiChE compounds on peripheral and central nervous systems with subsequent accumulation of acetylcholine and overstimulation of synapses, hypoxia, and hemodynamic instability and hypoperfusion due to arrhythmia and hypotension (Liu et al., 2008; Kozacı et al., 2012).

In this study, PSS grades significantly correlated with the delay in hospital arrival and length of hospital stay periods as well as vital signs at the time of admission.

Similarly, Sam et al. (2009) have concluded that the delay in prehospitalization time can influence the severity of AntiChE poisoning as evidenced by a significant linear correlation between the prehospitalization period and PSS grades. On the other side, Akdur et al. (2010) and Azab (2015) have found insignificant correlation and no significant difference, respectively, between pre-hospitalization period and PSS grades.

In addition, Rehiman et al. (2008) and Azab (2015) have reported a significant positive association and a significant difference, respectively, between the severity of poisoning grades and the length of hospital stay, thus the hospitalization period being higher in grade 3 intoxicated patients on admission. On the contrary, Sam et al. (2009) have observed insignificant correlation between PSS and the hospitalization period.

Several authors have suggested that criteria such as cardio-respiratory rates can assess severity of AntichE intoxication and help in predicting possible outcome (Senanayake et al., 1993).

Also, the study of Yu et al. (2012) has showed significant association between altered vital signs and poisoning-related severity. The prior authors stated that vital signs may play an important role in diagnosis, assessing, and prediction of the clinical severity and outcome among poisoned patients. Unlikely, Davies et al. (2008) has proclaimed that the heart rate is of little value in predicting the outcome of AntiChE poisoning and weakly contributes to the predictive value of the PSS.

The present results demonstrated significant correlation between the GCS scores and the delay in hospital arrival and length of hospital stay periods as well as vital signs at the time of admission.

Likewise, the study of Muley et al. (2014) has shown markedly lower GCS score in concomitant with prolonged time elapsed after exposure to AntiChE substances in patients needed mechanical ventilation on admission. Also, the sensitivity and specificity of GCS at the time of admission to the medical facilities significantly contribute in predicting severity and outcome in dimethoate intoxicated patients (Davies et al., 2008). Furthermore, the study of Muley et al. (2014) has demonstrated a significant negative association between the duration of hospital stay and the GCS scores. 
The current work illustrated significant correlation between the BuChE levels and the prehospitalization and hospitalization periods as well as vital signs at the time of admission. The same findings have been reported by Muley et al. (2014), wherein great reduction in serum BuChE levels and considerable delay in pre-hospitalization periods are noticed in AntiChE poisoned patients, in addition, a moderate negative correlation is also seen between serum BuChE levels and hospital stay.

In general, the more delay in prehospitalization period, the more severity of poisoning degree including alternations in vital signs and mental status, and the more increase in hospitalization period. These abnormalities may be due to delay in early therapeutic intervention that subsequently increases the initial serum level of AntiChE substances, leading to irreversible organ damage (Akdur et al., 2010).

In the present study, a significant difference was noticed on comparing the mean GCS scores with the PSS grades of the three intoxicated-groups besides there was also a significant negative correlation between the degree of severity of poisoning and GCS. These findings are in agreement with the result of Azab (2015) who observed a significant distinction on matching GCS scores between the PSS grades (The lower the GCS score, the higher the PSS grade) as well as with the reports of Sam et al. (2009) and Akdur et al. (2010) who found a significant negative correlation between these two variables owing to low GCS scores and high PSS grades among AntiChE intoxicated patients. This may be due to high lipophilic properties of OPC with their ease of blood-brain barrier penetration. Although CMC do not easily cross into the nervous system, but in massive poisonings, neurological dysfunctions may occur from hypoxic effects secondary to pulmonary toxicity and paralysis (Eddleston and Clark, 2011).

In the current study, there was a significant inverse correlation between the PSS grades and BuChE enzyme levels. This finding is in line with previous other reports which have described a significant negative correlation between the derangement of serum BuChE levels and/or erythrocytes cholinesterase activities and the severity of cholinergic toxidrome following AntiChE poisoning (Weissmann-Brenner et al., 2002; Rehiman et al., 2008; Soysal et al., 2011). Conversely to this, several other authors did not find a relationship between the PSS and the decrease in cholinesterase activities of AntiChE intoxicated cases (Sam et al., 2009; Akdur et al., 2010; Azab, 2015). This negative correlation is due to that AntiChE chemicals mainly cause their toxicity through inhibition of the activities of different cholinesterase enzymes (Lotti, 2002; Erdman, 2004).

In the current study, the chief detected abnormal biochemical findings in some patients were low $\mathrm{pH}$ of acidbase balance (metabolic acidosis), hypernatremia, hypokalemia, and hyperglycaemia. There were significant differences between AntiChE-group at the time of admission and HC-group regarding the mean levels of these biochemical parameters; however, there were insignificant differences among both groups at discharge. These parameters showed different correlation coefficient significances with PSS grades, GCS scores, and BuChE levels.

Metabolic acidosis is a relatively 
common complication in AntiChE poisoning and can occur due to either hypoventilation or hypotension (Erdman, 2004; Bhattacharyya et al., 2011). Also, several researchers have determined occurrence of electrolytes abnormalities including hypernatremia (Laudari et al., 2014) and hypokalemia (Tsai et al., 2007; Laudari et al., 2014; Abd El Al et al., 2016) in AntiChE poisoned cases. These changes in electrolytes levels may be explained by excess cholinergic stimulation and/or severe vomiting and diarrhea (Eddleston and Clark, 2011).

Additionally, several investigators proclaimed that hyperglycaemia is a common complication and a clinically significant finding accompanied with AntiChE poisoning (Sungur and Güven, 2001; Sahin et al., 2002; Yurumez et al., 2007). This increase in blood glucose levels may be due to secondary pancreatic injury (Singh et al., 2007) or release of catecholamines from the adrenal medulla that subsequently stimulate glycogenolysis and induce hyperglycemia (Yurumez et al., 2007; Eddleston and Clark, 2011).

Liu et al. (2008) and Moon et al. (2016), respectively, have found that the low $\mathrm{pH}$ and hyperglycaemia positively correlated with the clinical severity of acute AntiChE poisoning on admission, which are in agreement with the present findings.

The present study demonstrated alternations in the assayed enzymatic biomarkers values of poisoned cases. Low BuChE levels were seen in all cases, whereas increased serum levels of CK-MB, cTnI, amylase, lipase, AST, ALT, BLU, and CRE were detected in some cases.

In general, BuChE levels typically decline more quickly after exposure to
AntiChE chemicals; therefore, its activity is routinely assayed for the early diagnosis and continuous monitoring of poisonings by these compounds (Lotti, 2002; Erdman, 2004; Rehiman et al., 2008). In this work, poisonings by AntiChE chemicals were fundamentally associated with a significant reduction in BuChE activity, which is similar to numerous other studies conducted in Egypt (Ibrahim et al., 2011; Abdel Haleem et al., 2012; Abd El Al et al., 2016) and other countries (Rehiman et al., 2008; Sam et al., 2009; Akdur et al., 2010; Soysal et al., 2011).

Also, comparison between the mean serum BuChE level at the time of discharge and that at admission revealed significant increase, but still significantly lower than HC-group value. This might be attributed to the early arrival of patients to the hospital as well as early administration of the antidotes such as atropine and cholinesterase reactivator oxime (obidoxime chloride). In untreated patients, normalization of inhibited BuChE takes about 4-6 weeks to return to pre-exposure levels and its activity increases by about $25-30 \%$ in the first 7-10 days (Eddleston and Clark, 2011).

Elevations of serum CK-MB and cTnI levels were noticed in some patients and there were significant increases in their mean serum values of AntiChE-group at the time of admission as compared to the control values. However, at the time of discharge, their levels were markedly decreased and became non-significantly different from the HC-GP. These findings are compatible with many other publications illustrating augmentation of CK-MB and/or cTnI cardiac enzymes levels in AntiChE 
intoxicated patients (Wanf et al., 2011; Cha et al., 2014) with statistically significant differences between poisoned and control groups (Wanf et al., 2011).

The present study showed significant positive correlations between the changes in CK-MB and cTnI cardiac enzymes and the severity of poisoning, which have also been reported by others (Kharoub and Elsharkawy, 2008; Wanf et al., 2011; Cha et al., 2014).

These alterations in cardiac enzymes levels may be attributed to the direct myocardial cell damage or ischemic injury, resulting in leakage of these enzymes into blood (Anand et al., 2009; Cha et al., 2014).

In the current work, increases in serum amylase and lipase were noticed in several patients of AntiChE-group at the time of admission. These pancreatic enzymes showed significant differences when compared with HC-group. Analogously, several other literatures have shown transient development of hyperamylasemia and hyperlipasemia in patients with AntiChE poisoning (Sahin et al., 2002; Singh et al., 2007; Yurumez et al., 2007; Abd El Al et al., 2016).

Also, the present results illustrated significant positive association between the PSS grades and both lipase and amylase. These findings are in harmony with several other studies clarifying that elevation in serum amylase and lipase levels closely related to the clinical severity and the presence of different complications such as shock and respiratory failure (Kozaci et al., 2012). In contrast, the work of Singh et al. (2007) has not detected any correlation between hyperamylasemia and poisoning severity.

The possible pathogenetic mechanisms of AntiChE-induced pancreatic insult are multifactorial, including excessive release of acetylcholine from pancreatic tissue with subsequent prolonged hyperstimulation of pancreatic acinar cells (Harputluoglu et al., 2007) or development of pancreatitis secondary to increase pancreatic sensitivity and secretions in response to acetylcholine, acinar cell vacuolation, and edema (Brahmi et al., 2005).

The present study depicted elevations of serum AST and ALT levels in some intoxicated cases on admission and these changes were found to be statistically significant when compared with HC-group. These results are in concordant with many other literatures clarifying abnormal increases in liver enzymes among AntiChE intoxicated patients (Sahin et al., 2002; Tsai et al., 2007; Yurumez et al., 2007; Awad et al., 2014). On the contrary, other Egyptian study has shown insignificant differences between AntiChE poisoned patients and healthy control individuals regarding the serum enzyme levels of AST and ALT (Abdel Haleem et al., 2012).

In the present study, there was significant positive correlation between elevated liver enzymes and the severity of poisoning. Also, other results of acute AntiChE poisoning displaying higher liver enzymes levels as potential risk factors and could be used as a predictive value for poisoning severity (Ram et al., 1991).

Multiple mechanisms such as exposure time and dose (Erdman, 2004), formation and accumulation of more toxic metabolites than the parent AntiChE compounds, leading to P450 inactivation (Ncibi et al. 2008), or oxidative stress in hepatocellular mitochondria, resulting in disturbance 
of oxidants/antioxidants balance

(Yurumez et al., 2007) may contribute in the development of AntiChE-induced hepatotoxicity with subsequent elevation of blood liver enzymes.

The current study showed augmentation of BLU and CRE levels in some intoxicated cases on admission as well as a significant positive correlation between the severity of poisoning and kidney function tests. These abnormalities are in harmonization with several other reports elucidating impairment of renal function in AntiChE poisoned patients, which is widely correlated with the severity of poisoning (Agostini and Bianchin, 2003; Abd El Al et al., 2016). In contrast, other authors have documented insignificant variations between AntiChE poisoned cases and healthy control persons concerning the serum enzyme levels of blood urea nitrogen and creatinine (Abdel Haleem et al., 2012). Acute nephrotoxicity after AntiChE poisoning appears to result from proximal renal tubular damage secondary to elevation of BuChE levels in renal tissue, high intratubular concentrations of AntiChE chemicals, rhabdomyolysis, and/or hypovolaemia from dehydration (Agostini and Bianchin, 2003; Yurumez et al., 2007).

The present work declared different significant correlation between $\mathrm{BuChE}$ levels and all investigated biochemical and enzymatic biomarkers of AntiChEgroups on admission. As noticed in the previous studies, severe perturbations of these laboratory parameters are strongly accompanied with markedly low BuChE levels in AntiChE poisoned patients.

CONCLUSION:

Acute AntiChE poisoning has induced various vital signs dysfunctions, metabolic derangements, and serious toxic effects on cardiac, pancreatic, hepatic, and renal organs as evidenced by elevations of their corresponding serum enzymatic biomarkers. Although the PSS grades, GCS scores, and BuChE levels at presentation appear similarly effective clinical indices for assessing and predicting the severity of AntiChE poisoning as manifested by their significant correlations with the investigated clinical and laboratory parameters as well as between each other, the efficacy of PSS outperform the GCS and BuChE effectiveness. The patients with evidence of moderate and severe degree of poisoning need to be monitored closely.

\section{REFERENCES}

Abd El Al, A.H., Fawzi, M.M., ALKhafif, M.A., and El-Zemaity, M.E. (2016): Epidemiological study of organophosphorus compounds insecticides types related to acutely intoxicated patients presented to Poison Control Center (PCC-ASU)-Egypt. IOSR-JESTFT, 10:72-78.

Abdel Haleem, Z.A., Abdel kareem, M.A., and Ahmed, E.K. (2012): Beta Glucoronidase as a Simple Biomarker of Acute Organophosphate Poisoning in Human. Egypt J Forensic Sci Appli Toxicol, 12:157-168.

Adams, J.E., Schechtman, K.B., Landt, Y., Ladenson, J.H., and Jaffe, A.S. (1994): Comparable detection of acute myocardial infarction by creatine kinase $\mathrm{MB}$ isoenzyme and cardiac troponin I. Clin Chem, 40:1291-1295.

Agostini, M. and Bianchin, A. (2003): 
Acute renal failure from organophosphate poisoning: a case of success with haemofiltration. Hum Exp Toxicol, 22:165-167.

Akdur, O., Durukan, P., Ozkan, S., Avsarogullari, L., Vardar, A., Kavalci, C., and Ikizceli, I. (2010): Poisoning severity score, Glasgow coma scale, corrected QT interval in acute organophosphate poisoning. Hum Exp Toxicol, 29:419-425.

Al-Attar, A.M. (2010): Physiological and histopathological investigations on the effects of $\alpha$-lipoic acid in rats exposed to malathion. $\mathbf{J}$ Biomed Biotechnol, 203503:1-8.

Anand, S., Singh, S., Nahar Saikia, U., Bhalla, A., Paul Sharma, Y., and Singh, D. (2009): Cardiac abnormalities in acute organophosphate poisoning. Clin Toxicol (Phila), 47:230-235.

Aslan, S., Cakir, Z., Emet, M., Serinken, M., Karcioglu, O., Kandis, H., and Uzkeser, M. (2011): Acute abdomen associated with organophosphate poisoning. J Emerg Med, 41:507-512.

Awad, O.M., El-Fiki, S.A., AbouShanab, R.A.I., Hassanin, N.M.A., and Abd El Rahman, R. (2014): Influence of exposure to pesticides on liver enzymes and cholinesterase levels in male agriculture workers. Global Nest Journal, 16:1006-1015.

Azab, G.B. (2015): Poisoning Severity Score and Glasgow Coma Scale Are Tools for Estimating Severity and Prognosis of Patients with Acute Carbamate Poisoning. Egypt J Forensic Sci Appli Toxicol, 2:159-172.

Baydin, A., Aygun, D., Yazici, M., Karatas, A., Deniz, T., and Yardan, T. (2007): Is there a relationship between the blood cholinesterase and QTc interval in the patients with acute organophosphate poisoning? Int J Clin Pract, 61:927-930.

Bhattacharyya, K., Phaujdar, S., Sarkar, R., and Mullick, O.S. (2011): Serum creatine phosphokinase: a probable marker of severity in organophosphorus poisoning. Toxicol Int, 18:117-123.

Brahmi, N., Blel, Y., Kouraichi, N., Abidi, N., Thabet, H., and Amamou, M. (2005): Acute pancreatitis subsequent to voluntary methomyl and dichlorvos intoxication. Pancreas, 31:424-427.

Cha, Y.S., Kim, H., Go, J., Kim, T.H., Kim, O.H., Cha, K.C., Lee, K.H., and Hwang, S.O. (2014): Features of myocardial injury in severe organophosphate poisoning. Clin Toxicol (Phila), 52:873-879.

Colović, M.B., Krstić, D.Z., Lazarević-Pašti, T.D., Bondžić, A.M., and Vasić, V.M. (2013): Acetylcholinesterase inhibitors: pharmacology and toxicology. Curr Neuropharmacol, 11:315-335.

Costa, L.G. (2006): Current issues in organophosphate toxicology. Clin Chim Acta, 366:1-13.

Davies, J.O., Eddleston, M., and Buckley, N.A. (2008): Predicting outcome in acute organophosphorus poisoning with a poison severity score or the Glasgow coma scale. QJM, 101:371-379.

Eddleston, M. and Clark, R.F. (2011): Insecticides: Organic Phosphorus Compounds and Carbamates. In: Goldfrank's Toxicologic Emergencies. Nelson, L.S.; Lewin, N.A.; Howland, M.A.; et al. \{Eds. $\} .9^{\text {th }}$ ed., Ch. 113, McGraw-Hill Companies, New 
York, pp:1450-1466.

Eddleston, M., Dawson, A., Karalliedde, L., Dissanayake, W., Hittarage, A., Azher, S., and Buckley, N.A. (2004): Early management after self-poisoning with an organophosphorus or carbamate pesticide-a treatment protocol for junior doctors. Crit Care, 8:R391-R397.

Eddleston, M., Mohamed, F., Davies, J.O., Eyer, P., Worek, F., Sheriff, M.H., and Buckley, N.A. (2006): Respiratory failure in acute organophosphorus pesticide selfpoisoning. QJM, 99:513-522.

Erdman, A.R. (2004): Insecticides. In: Medical toxicology. Dart, R.C.; Caravati, E.M.; McGuigan, M.A.; et al. (Eds.). $3^{\text {rd }}$ ed., Lippincott Williams \& Wilkins, Philadelphia, pp:1475-1496.

Exner, C.J. and Ayala, G.U. (2009): Organophosphate and carbamate intoxication in La Paz, Bolivia. J Emerg Med, 36:348-352.

Foo, A.Y. and Bais, R. (1998): Amylase measurement with 2chloro 4nitrophenyl nmaltrotrioside as substrate. Clin Chim Acta, 272:137-147.

Gerhardt, W. and Waldenström, J. (1979): Creatine kinase B-subunit activity in serum after immunoinhibition of M-subunit activity. Clin Chem, 25:1274-1280.

Goldsmith, M., Ashani, Y., Margalit, R., Nyska, A., Mirelman, D., and Tawfik, D.S. (2016): A new postintoxication treatment of paraoxon and parathion poisonings using an evolved PON1 variant and recombinant GOT1. Chem. Biol Interact, 259:242-251.

Gündüz, E., Dursun, R., Icer, M., Zengin, Y., Güllü, M.N., Durgun, H.M., and Gokalp, O. (2015):
Factors affecting mortality in patients with organophosphate poisoning. J Pak Med Assoc, 65:967-972.

Harputluoglu, M.M., Demitel, U., Alan, H., Ateş, F., Aladă̆, M., Karincaoğlu, M., and Hilmioğlu, F. (2007): Pancreatic pseudocyst development due to organophosphate poisoning. Turk J Gasteroentterol, 18:122-125.

Ibrahim, M.A., El Masry, M.K., Moustafa, A.A., Hagras, A.M., and Ali, N.M. (2011): Comparison of the accuracy of two scoring systems in predicting the outcome of organophosphate intoxicated patients admitted to intensive care unit (ICU). Egypt J Forensic Sci, 1:41-47.

Kara, I.H., Güloğlu, C., Karabulut, A., and Orak, M. (2002): Sociodemographic, clinical, and laboratory features of cases of organic phosphorus intoxication who attended the Emergency Department in the Southeast Anatolian Region of Turkey. Environ Res, 88:82-88.

Karki, P., Ansari, J.A., Bhandari, S., and Koirala, S. (2004): Cardiac and electrophysiological manifestations of acute organophosphate poisoning. Singapore Med J, 45:385-389.

Kharoub, M.A. and Elsharkawy, S.A. (2008): Cardiotoxicity of acute organophosphate poisoning. Benha M J, 25:9-24.

Kozacı, N., Gokel, Y., Acıkalın, A., and Satar, S. (2012): Factors affecting the prognosis in acute insecticide intoxications containing organic phosphorus. J Academic Emerg Med., 11:93-97.

Kumar, S.V., Fareedullah, M., Sudhakar, Y., Venkateswarlu1, 
E., and Kumar, A. (2010): Current review on organophosphorus poisoning. Arch Appl Sci Res, 2:199-215.

Kunsst, A. (1994): Determination of glucose level. In: Methods of Enzymatic Analysis. Bergmeyer, H.U. (Ed.). $3^{\text {rd }}$ ed, Academic Press. New York, Volume (2), pp:163172.

Lamb, E.J. and Price, C.P. (2008): Creatinine, urea and uric acid. In: Tietz fundamentals of clinical chemistry. Burtis, C.A.; Ashwood, E.R.; and Bruns, D.E. (Eds.). $6^{\text {th }}$ ed., $\quad$ Saunders Elsevier; Philadelphia, pp:363-372.

Laudari, S., Patowary, B.S., Sharma, S.K., Dhungel, S., Kumudini, K., Bhattacharya, R., Guru-Prasad, S., and Gangapatnam, S. (2014): Cardiovascular Effects of Acute Organophosphate Poisoning. Asia Pac J Med Toxicol, 3:64-67.

Lee, F.Y., Chen, W.K., Lin, C.L., Lai, C.Y., Wu, Y.S., Lin, I.C., Kao, and C.H. (2015): Organophosphate poisoning and subsequent acute kidney injury risk: a nationwide population-based cohort study. Medicine (Baltimore), 94:e2107.

Liu, J.H., Chou, C.Y., Liu, Y.L., Liao, P.Y., Lin, P.W., Lin, H.H., and Yang, Y.F. (2008): Acid-base interpretation can be the predictor of outcome among patients with acute organophosphate poisoning before hospitalization. Am. J Emerg Med, 26:24-30.

Lorentz, K. (1998): Lipase. In: Clinical Laboratory Diagnostic: use and assessment of clinical laboratory results. Thomas, L. (Ed.); $\quad 1^{\text {st }} \quad$ ed., TH-Books Verlagsgesellschaft, Frankfurt, pp: 95-97.
Lotti, M. (2002): Clinical Toxicology of Anticholinesterase Agents in Humans. In: Handbook of Pesticide Toxicology. Krieger, R. (Ed.). $2^{\text {nd }}$ ed., Ch.33, Academic Press, San Diego, pp:1043-1085.

Mansour, S. and Gamalludin, $\mathbf{H}$. (2008): Pattern of human acute intoxication in Egypt with special concern to pesticide poisoning and farmers' attitude regarding use of these substances. Toxicol Lett, 180:S32-S246.

Moon, J.M., Chun, B.J., and Cho, Y.S. (2016): Hyperglycemia at presentation is associated with in hospital mortality in non-diabetic patient with organophosphate poisoning. Clin Toxicol (Phila), 54:252-258.

Muley, A., Shah, C., Lakhani, J., Bapna, M., and Mehta, J. (2014): To identify morbidity and mortality predictors in acute organophosphate poisoning. Indian J Crit Care Med, 18:297-300.

Ncibi, S., Othman, M.B., Akacha, A., Krifi, M.N., and Zourgui, L. (2008): Opuntia ficus indica extract protects against chlorpyrifosinduced damage on mice liver. Food Chem Toxicol, 46:797-802.

Nelson, L.S., Lewin, N.A., Howland M.A., Hoffman, R.S., Goldfrank, L.R., and Flomenbaum, N.E. \{Eds.\} (2011): Initial Evaluation of the Patient: Vital Signs and Toxic Syndromes. In: Goldfrank's Toxicologic Emergencies. 9 ${ }^{\text {th }}$ ed., Ch.3, McGraw-Hill Companies, New York, pp:33-36.

Ozer, C., Kuvandik, G., Gokel, Y., Duru, M., and Helvaci, M.R. (2007): Clinical presentation and laboratory findings of organic phosphorus poisoning. Adv Ther, 24:1321-1329. 
Persson, H.E., Sjöberg, G.K., Haines, J.A., and Pronczuk de Garbino, J. (1998): Poisoning severity score. Grading of acute poisoning. J Toxicol Clin Toxicol, 36:205-213.

Pincus, M,R., Tierno, P., and Dufour, D.R. (2007): Evaluation of Liver Function. In: Henry's Clinical Diagnosis and Management by Laboratory Methods. McPherson, R.A. and Pincus, M.R. (Eds.). Ch.21, $21^{\text {st }}$ ed., Saunders Company, Philadelphia, U.S.A., pp:457-472.

Pincus, M.R. and Lifshitz, M.S. (2007): Evaluation of Renal Function, Water, Electrolytes and Acid-Base Balance. In: Henry's Clinical Diagnosis and Management by Laboratory Methods. McPherson, R.A. and Pincus, M.R. (Eds.). Ch.14, $21^{\text {st }}$ ed., Saunders Company, Philadelphia, U.S.A., pp:360-376.

Ram, J.S., Kumar, S.S., Jayarajan, A., and Kuppuswamy, G. (1991): Continuous infusion of high doses of atropine in the management of organophosphorus compound poisoning. J Assoc Physicians India, 39:190-193.

Rehiman, S., Lohani, S.P., and Bhattarai, M.D. (2008): Correlation of Serum Cholinesterase Level, Clinical Score at Presentation and Severity of Organophosphorous Poisoning. J Nepal Med Assoc, 47:47-52.

Rubinshtein, R., Bar-Meir, E., Grubstein, A., and Bitterman, $\mathbf{H}$. (2002): Early onset of ventricular tachydsrhythmias in organophosphate intoxication. Isr Med Assoc J, 4:63-64.

Saadeh, A.M., al-Ali, M.K., Farsakh, N.A., and Ghani, M.A. (1996): Clinical and sociodemographic features of acute carbamate and organophosphate poisoning: a study of 70 adult patients in north Jordan. J Toxicol Clin Toxicol, 34:45-51.

Sahin, I., Onbasi, K., Sahin, H., Karakaya, C., Ustun, Y., and Noyan, T. (2002): The prevalence of pancreatitis in organophosphate poisoning. Hum Exp Toxicol, 21:175-177.

Sam, K.G., Kondabolu, K., Pati, D., Kamath, A., Pradeep Kumar, G., and Rao, P.G. (2009): Poisoning severity score, APACHE II and GCS: effective clinical indices for estimating severity and predicting outcome of acute organophosphorus and carbamate poisoning. J Forensic Leg Med, 16:239-247.

Satoh, T. (2006): Global Epidemiology of Organophosphate and Carbamate Poisonings. In: Toxicology of Organophosphate and Carbamate Compounds. Gupta RC (Ed.). Ch.8, Elsevier Academic Press, London, pp.89-100.

Scott, M.G., LeGrys, M.T., and Klutts, J.S. (2006): Electrolytes and Blood Gases. In: Tietz Textbook of Clinical Chemistry and Molecular Diagnostics. Burtis CA, Ashwood ER and Bruns DE (Eds.). Ch.27, $4^{\text {th }}$ ed., Elsevier Saunders, Philadelphia, pp:9831018.

Senanayake, N., de Silva, H.J., and Karalliedde, L. (1993): A scale to assess severity in organophosphorus intoxication: POP scale. Hum Exp Toxicol, 12:297-299.

Singh, S., Bhardwaj, U., Verma, S.K., Bhalla, A., and Gill, K. (2007): Hyperamylasemia and acute pancreatitis following anticholinesterase poisoning. Hum Exp Toxicol, 26:467-471. 
Singh, S., Kumar, V., Thakur, S., Banerjee, B.D., Chandna, S., Rautela, R.S., Grover, S.S., Rawat, D.S., Pasha, S.T., Jain, S.K., Ichhpujani, R.L., and Rai, A. (2011): DNA damage and cholinesterase activity in occupational workers exposed to pesticides. Environ Toxicol Pharmacol, 31:278-285.

Soltaninejad, K., Faryadi, M., and Sardari, F. (2007): Acute pesticide poisoning related deaths in Tehran during the period 2003-2004. J Foren Legal Med, 14:352-354.

Soysal, D., Karakuş, V., Soysal, A., Tatar, E., Yıldız, B., and Şimşek, H. (2011): Evaluation of cases with acute organophosphate pesticide poisoning presenting at a tertiary training hospital emergency department: intoxication or suicide? JAEM, 10:156-160.

Sungur, M. and Güven, M. (2001): Intensive care management of organophosphate insecticide poisoning. Crit Care, 5:211-215.

Talaie, H., Owliaey, H., Pajoumand, A., Gholaminejad, M., and Mehrpour, O. (2012): Temperature changes among organophosphate poisoned patients, Tehran-Iran. Daru, 20:52.

Titlić, M., Josipović-Jelić, Z., and Punda, A. (2008): Headache caused by pesticides--a review of the literature. Acta Med Croatica, 62:233-236.

Tsai, J., Sheu, C., Cheng, M., Hung JY, Wang, C.S., Chong, I.W., Huang, M.S., and Hwang, J.J. (2007):

Organophosphate poisoning: 10 years of experience in Southern Taiwan. Kaohsiung $\mathbf{J}$
Med Sci, 23:112-119.

Vijayakumar, S., Fareedullah, M., Kumar, A.E., and Mohan Rao, K. (2011): A prospective study on electrocardiographic findings of patients with organophosphorus poisoning. Cardiovasc Toxicol, 11:113-117.

Wanf, Z.X., Wang, C.X., Liu, S.H., Shi, J.H., and Tu, Y.Y. (2011): Variation and clinical significance of serum CTnT and CK-MB in patients with acute organophosphorus pesticid poisoning. Xi Bao Yu Fen Zi Mian Yi Xue Za Zhi, 27:90-91.

Weissmann-Brenner, A., Aviv-Vidan, A., and Hourvitz, A. (2002): Organophosphate poisoning: A multihospital survey. IMAJ, 4:573576.

Whittaker, M., Britten, J.J., and Dawson, P.J. (1983): Comparison of a commercially available assay system with two reference methods for the determination of plasma cholinesterase variants. Clin Chem, 29:1746-1751.

Yu, J.H., Weng, Y.M., Chen, K.F., Chen, S.Y., and Lin, C.C. (2012): Triage vital signs predict inhospital mortality among emergency department patients with acute poisoning: a case control study. BMC Health Serv Res, 12:262-269.

Yurumez, Y., Durukan, P., Yavuz, Y., Ikizceli, I., Avsarogullari, L., Ozkan, S., Akdur, O., and Ozdemir, C. (2007): Acute organophosphate poisoning in university hospital emergency room patients. Intern Med, 46:965 -969. 


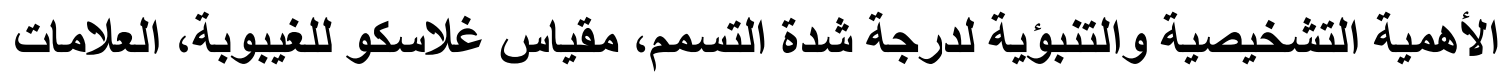

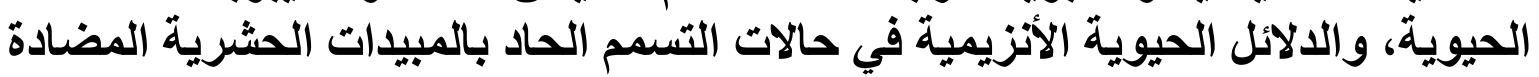 لإنزيم الكولينستريز
}

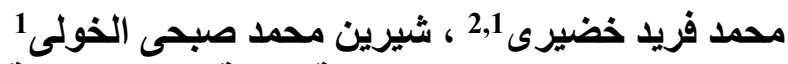

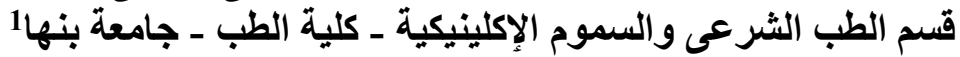

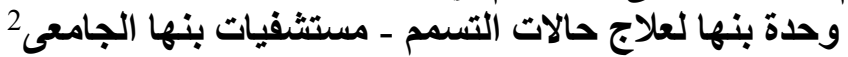

المقدمة : مركبات الفوسفات العضوية و الكربامات هي مبيد العربى اتلبـ الآفات المضـادة لعمل إنزيم الكولينستريز

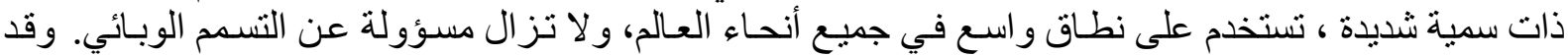

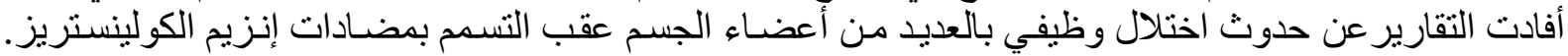

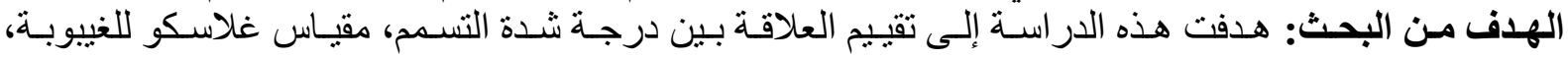

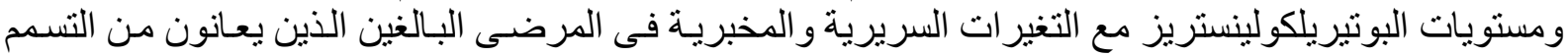

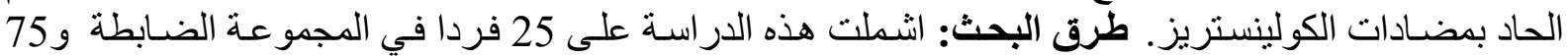

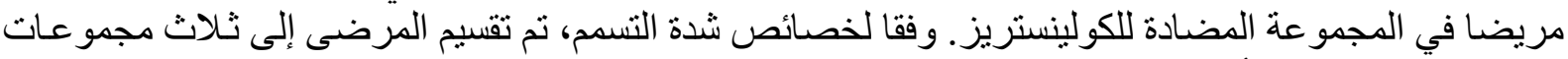

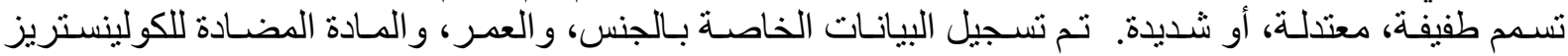

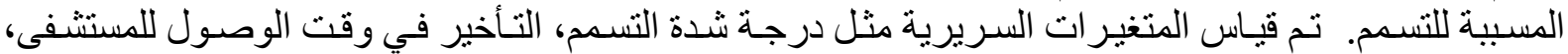

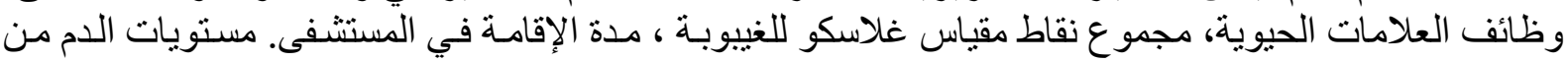

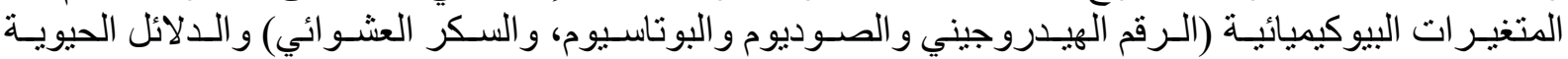

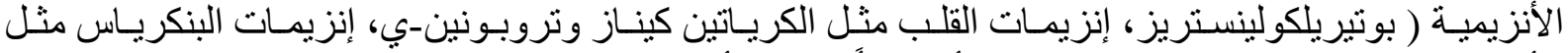

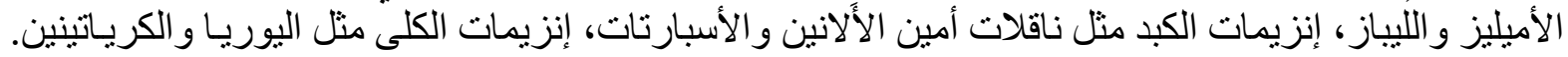

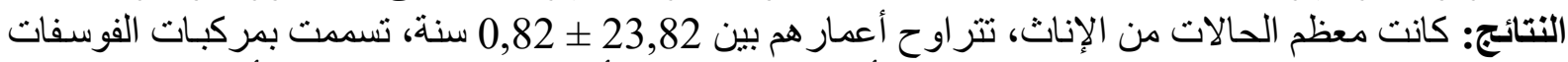

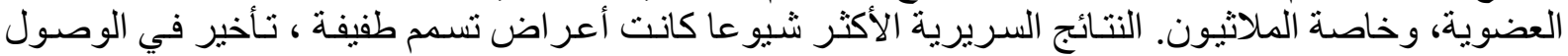
للمستشفى لمدة 2 سـاعة ، ومستوى النعاس على مقياس غلاسكو للغيبوبـة، ومدة اقامـة بالمستشفى قصبيرة نسبيا

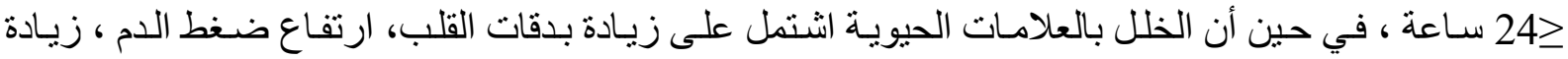

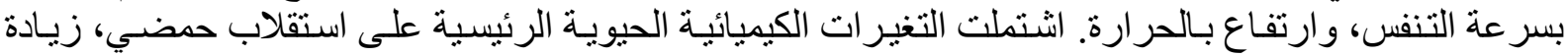

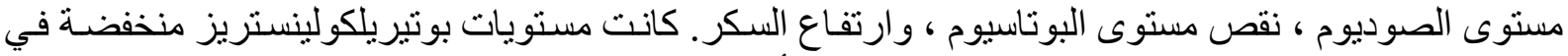

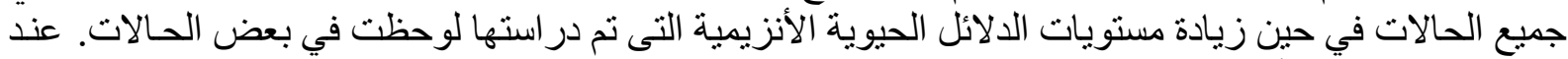

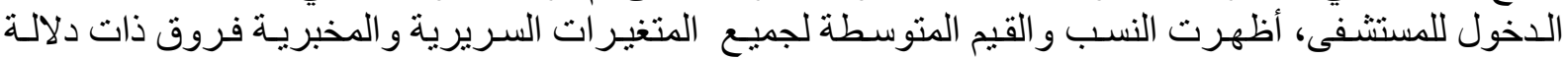

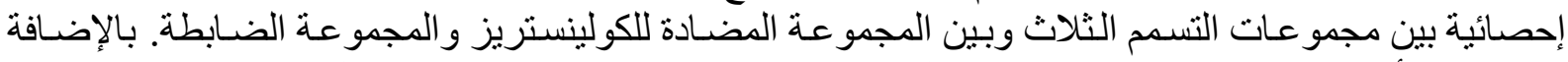

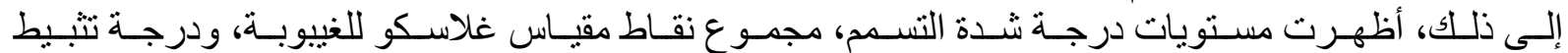

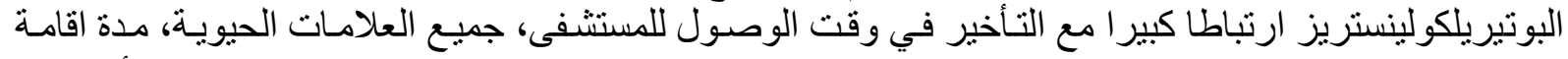

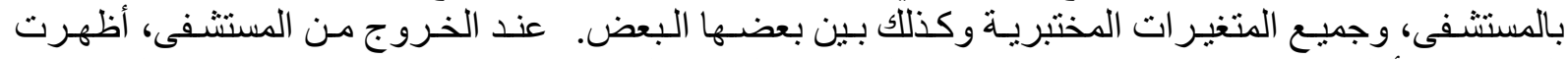

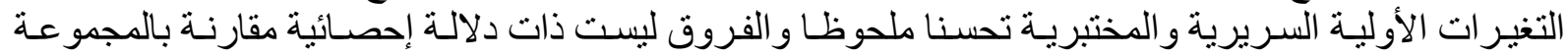

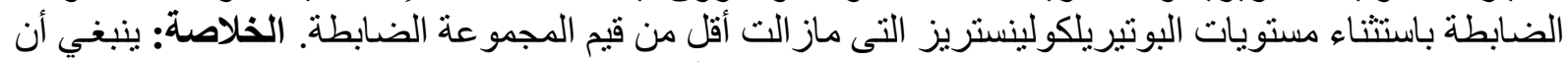

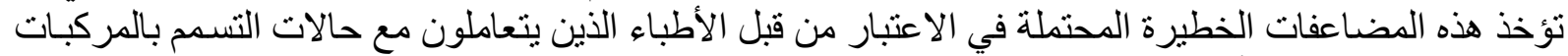

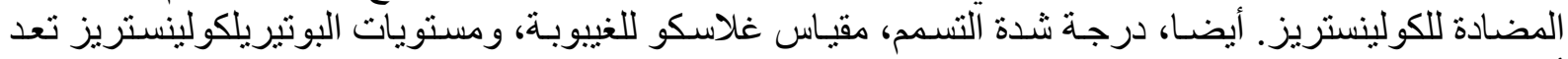

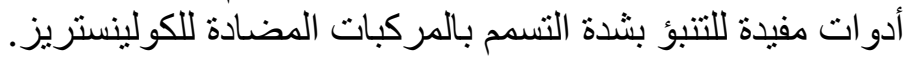

